\title{
On the Nonsymmetric Longer Queue Model: Joint Distribution, Asymptotic Properties, and Heavy Traffic Limits
}

\author{
Charles Knessl ${ }^{1}$ and Haishen Yao ${ }^{2}$ \\ ${ }^{1}$ Department of Mathematics, Statistics and Computer Science, University of Illinois at Chicago, Chicago, IL 60607-7045, USA \\ ${ }^{2}$ Department of Mathematics and Computer Science, QCC, The City University of New York, 222-05 56th Avenue, Bayside, \\ NY 11364, USA
}

Correspondence should be addressed to Haishen Yao; hyao@qcc.cuny.edu

Received 28 January 2013; Accepted 8 April 2013

Academic Editor: Khosrow Moshirvaziri

Copyright (C) 2013 C. Knessl and H. Yao. This is an open access article distributed under the Creative Commons Attribution License, which permits unrestricted use, distribution, and reproduction in any medium, provided the original work is properly cited.

We consider two parallel queues, each with independent Poisson arrival rates, that are tended by a single server. The exponential server devotes all of its capacity to the longer of the queues. If both queues are of equal length, the server devotes $\nu$ of its capacity to the first queue and the remaining $1-v$ to the second. We obtain exact integral representations for the joint probability distribution of the number of customers in this two-node network. Then we evaluate this distribution in various asymptotic limits, such as large numbers of customers in either/both of the queues, light traffic where arrivals are infrequent, and heavy traffic where the system is nearly unstable.

\section{Introduction}

We consider a nonsymmetric version of the longer queue model. Here there are two parallel queues, each fed by a Poisson arrival stream. There is but a single server who tends to the longer of the two queues. If the number of customers in each queue is the same, then the server devotes $v$ of its capacity to the first queue and $1-v$ to the second queue, with $0<v<1$. We let $N_{1}\left(N_{2}\right)$ denote the number of customers in the first (second) queue, the two arrival rates are $\lambda_{1}$ and $\lambda_{2}$, and the server works at rate $\mu$. Note that the total number of customers, $N_{1}+N_{2}$, in the two-node network behaves as the standard $M / M / 1$ model, so in the steady state we have the geometric distribution Prob $\left[N_{1}+\right.$ $\left.N_{2}=J\right]=\left(1-\rho_{1}-\rho_{2}\right)\left(\rho_{1}+\rho_{2}\right)^{J}$, where $\rho_{1}=\lambda_{1} / \mu, \rho_{2}=$ $\lambda_{2} / \mu$, assuming the stability condition $\rho_{1}+\rho_{2}<1$. The "symmetric case" corresponds to $\lambda_{1}=\lambda_{2}$ (thus $\rho_{1}=\rho_{2}$ ) and $v=1 / 2$, and this was analyzed in detail by Flatto [1].

Such models were proposed by Zheng and Zipkin [2] to study problems in inventory control. In [2] finite capacities were assumed in the two queues, and the authors studied numerically the steady state probabilities $p(m, n)=$
$\operatorname{Prob}\left[N_{1}=m, N_{2}=n\right]$, in terms of the capacity size and also for different service disciplines, such as the longer queue (LQ) discipline here, and also the first-come-firstserved discipline.

In [1] the author used two-dimensional generating functions and analyticity arguments and obtained explicit expressions for $p(m, n)$, in the symmetric case, as contour integrals. Then asymptotic results were derived for the joint distribution $p(m, n)$, as $m$ and/or $n$ becomes large, and also the marginal tails and various conditional limit laws were obtained. In this paper we generalize some of the results of [1] to the nonsymmetric model, and we will show that now many of the asymptotic results become quite different. As in [1] we assume that the model is preemptive, so if $N_{1}=N_{2}$ (and thus the server works on both queues) and a new arrival occurs to the first queue, then the server switches immediately all its capacity to the first queue. The more difficult nonpreemptive version of the LQ model was studied by Cohen [3], in the case of Poisson arrivals and general service times. The problem is reduced in [3], using generating functions, to a functional equation which is furthermore converted to a Riemann-Hilbert boundary value problem. 
The present problem corresponds to a random walk in the quarter plane (as $N_{1}, N_{2} \geqslant 0$ ), and other examples of such problems include shortest queue (SQ) problems [4-6], forkjoin models $[7,8]$, two coupled processors with generalized processor sharing [9-11], and two coupled tandem queues [12]. General techniques for solving such problems are discussed in [13-15] and they involve functional equations, analyticity arguments, and singular integral equation methods [16].

For the present model the analyticity arguments are fairly simple, and we focus mostly on the asymptotic properties of the solution. We will show that these asymptotics are quite different from those of the symmetric model in [1]. After obtaining exact integral representations for $p(m, n)$, and also the marginal probabilities $P(m)=\operatorname{Prob}\left[N_{1}=m\right]$ and $\mathscr{P}(n)=$ $\operatorname{Prob}\left[N_{2}=n\right]$, we asymptotically evaluate these integrals for $m$ and/or $n$ large. We use standard techniques, such as the Laplace method, saddle point method, singular analysis, and the Euler-MacLaurin formula. Good general references on the asymptotic evaluation of integrals and sums are the books [17-21].

In addition to this model being interesting on its own, many variants of shortest queue problems, such as ones with multiple servers and finite capacities, can be asymptotically reduced to LQ models of the type considered here (see $[22,23])$. For example, in [23] we showed that the finite capacity version of the standard symmetric SQ model (analyzed in [4,5]), where $N_{1}, N_{2} \leqslant K$ and $K$ is the capacity, asymptotically reduces to the symmetric LQ model in [1], if we consider the process $\left(K-N_{1}, K-N_{2}\right)$, which measures the number of spots available in the two waiting rooms. Then having a thorough understanding of the nonsymmetric LQ model and its asymptotics will allow us to analyze, at least in some asymptotic limits, nonsymmetric variants of SQ models.

The remainder of this paper is organized as follows. In Section 2 we summarize all of the main results, both exact and asymptotic. They are listed in Theorems 1-5, and some discussion/interpretation appears following each theorem. In Section 3 we briefly derive the exact expressions for $p(m, n)$; in Section 4 we derive asymptotic properties of $p(m, n)$ for $m, n$ large. In Section 5 we derive light traffic (where $\rho_{1}, \rho_{2} \rightarrow$ 0 ) and heavy traffic (where $\rho_{1}+\rho_{2} \uparrow 1$ ) results.

\section{Problem Statement and Summary of Results}

We let $\left(N_{1}, N_{2}\right)$ be the numbers of customers in the two parallel queues, and let $p(m, n)=\operatorname{Prob}\left[N_{1}=m, N_{2}=n\right]$ be the joint queue length distribution in the steady state. The two arrival rates are $\lambda_{1}$ and $\lambda_{2}$, the exponential server works at rate $\mu$, and $\rho_{1}=\lambda_{1} / \mu, \rho_{2}=\lambda_{2} / \mu$. If $N_{1}>N_{2}\left(N_{1}<N_{2}\right)$ the server works on the first (second) queue, but if $N_{1}=N_{2}$ the server works at rate $\mu \nu$ on the first queue and rate $\mu(1-\nu)$ on the second, with $0<v<1$. The symmetric case corresponds to $\lambda_{1}=\lambda_{2}$ and $v=1 / 2$. We henceforth assume the stability condition $\rho_{1}+\rho_{2}<1$.
In Figure 1 we sketch the transition rates for the random walk $\left(N_{1}, N_{2}\right)$, which illustrates the discontinuity along the "interface" $N_{1}=N_{2}$. The main balance equations are

$$
\begin{aligned}
\left(\rho_{1}+\right. & \left.\rho_{2}+1\right) p(m, n) \\
= & \rho_{1} p(m-1, n)+\rho_{2} p(m, n-1) \\
& \quad+p(m+1, n), \quad m>n+1, n \geqslant 1, \\
\left(\rho_{1}+\right. & \left.\rho_{2}+1\right) p(m, n) \\
= & \rho_{1} p(m-1, n)+\rho_{2} p(m, n-1) \\
& +p(m, n+1), \quad n>m+1, \quad m \geqslant 1 .
\end{aligned}
$$

We also have the following three interface equations:

$$
\begin{aligned}
\left(\rho_{1}+\right. & \left.\rho_{2}+1\right) p(n+1, n) \\
= & \rho_{1} p(n, n) \\
& +\rho_{2} p(n+1, n-1)+p(n+2, n) \\
& +(1-v) p(n+1, n+1), \quad n \geqslant 1, \\
\left(\rho_{1}+\right. & \left.\rho_{2}+1\right) p(n-1, n) \\
= & \rho_{1} p(n-2, n)+\rho_{2} p(n-1, n-1) \\
& +p(n-1, n+1)+v p(n, n), \quad n \geqslant 2, \\
\left(\rho_{1}+\rho_{2}\right. & +1) p(n, n) \\
= & \rho_{1} p(n-1, n)+\rho_{2} p(n, n-1) \\
& +p(n+1, n)+p(n, n+1), \quad n \geqslant 1,
\end{aligned}
$$

the two boundary conditions

$$
\begin{aligned}
& \left(\rho_{1}+\rho_{2}+1\right) p(m, 0) \\
& \quad=\rho_{1} p(m-1,0)+p(m+1,0), \quad m \geqslant 2, \\
& \left(\rho_{1}+\rho_{2}+1\right) p(0, n) \\
& \quad=\rho_{2} p(0, n-1)+p(0, n+1), \quad n \geqslant 2,
\end{aligned}
$$

and the corner conditions

$$
\begin{gathered}
\left(\rho_{1}+\rho_{2}+1\right) p(1,0)=\rho_{1} p(0,0)+(1-v) p(1,1)+p(2,0) \\
\left(\rho_{1}+\rho_{2}+1\right) p(0,1)=\rho_{2} p(0,0)+v p(1,1)+p(0,2) \\
\left(\rho_{1}+\rho_{2}\right) p(0,0)=p(1,0)+p(0,1) .
\end{gathered}
$$

The normalization is

$$
\begin{aligned}
\sum_{n=0}^{\infty} \sum_{m=0}^{\infty} p(m, n)= & \sum_{n=0}^{\infty} p(n, n)+\sum_{m>n} p(m, n) \\
& +\sum_{m<n} p(m, n)=1 .
\end{aligned}
$$




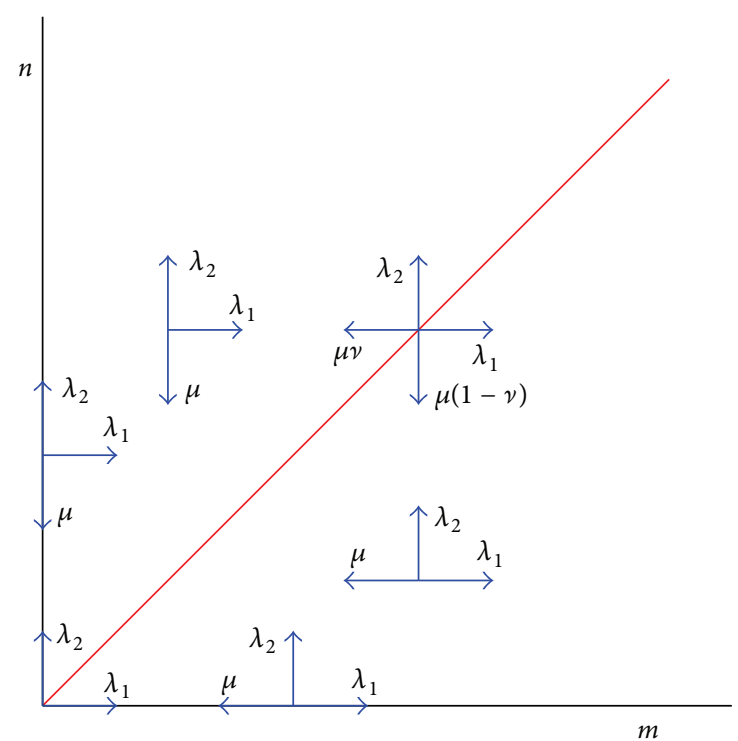

FIGURE 1: A sketch of the transition rates for the random walk.

Note that the elementary difference equations (6) and (7) may be solved immediately to obtain $p(m, 0)$ and $p(0, n)$, up to multiplicative constants. In Section 3 we analyze (1)-(9) to obtain $p(m, n)$ in the forms of contour integrals, which we summarize below.

Theorem 1. For $\rho_{1}+\rho_{2}<1$, the steady state distribution is as follows:

(i) $m>n$ :

$$
p(m, n)=\frac{1-\rho_{1}-\rho_{2}}{2 \pi i} \oint \frac{f(w)}{w^{n+1}\left[Z_{+}(w)\right]^{m-n}} d w,
$$

where the integral is over a small loop about $w=0$,

$$
\begin{gathered}
Z_{+}(w) \\
=\frac{1}{2 \rho_{1}}\left[\rho_{1}+\rho_{2}+1+\sqrt{\left(\rho_{1}+\rho_{2}+1\right)^{2}-4 \rho_{1}\left(\rho_{2} w+1\right)}\right], \\
f(w)=\frac{\operatorname{num}_{f}(w)}{\operatorname{den}(w)},
\end{gathered}
$$

$$
\begin{aligned}
\operatorname{num}_{f}(w) & \\
= & -2 \rho_{1} \rho_{2} w+\rho_{2}^{2}(1-v) \\
& +\rho_{1} \rho_{2}-\rho_{2}(1-v)+\left(\rho_{1}^{2}+\rho_{1}\right) \nu \\
& +\left[\rho_{2}-\left(\rho_{1}+\rho_{2}\right) \nu\right] \sqrt{\left(\rho_{1}+\rho_{2}+1\right)^{2}-4 \rho_{2}\left(\rho_{1} w+1\right)}
\end{aligned}
$$

$\operatorname{den}(w)$

$$
\begin{aligned}
= & {\left[\rho_{1} v+\rho_{2}(1-v)\right]\left(\rho_{1}+\rho_{2}+1\right) } \\
& -\rho_{1}\left(\rho_{2} w+v\right) \sqrt{\left(\rho_{1}+\rho_{2}+1\right)^{2}-4 \rho_{2}\left(\rho_{1} w+1\right)} \\
& -\rho_{2}\left(\rho_{1} w+1-v\right) \sqrt{\left(\rho_{1}+\rho_{2}+1\right)^{2}-4 \rho_{1}\left(\rho_{2} w+1\right)} .
\end{aligned}
$$

(ii) $m<n$ :

$$
\begin{aligned}
& p(m, n)= \frac{1-\rho_{1}-\rho_{2}}{2 \pi i} \oint \frac{g(w)}{w^{m+1}\left[Z_{+}^{*}(w)\right]^{n-m}} d w \\
& g(w)=\frac{\operatorname{num}_{g}(w)}{\operatorname{den}(w)} \\
& \operatorname{num}_{g}(w) \\
&=-2 \rho_{1} \rho_{2} w+\rho_{2}^{2}(1-v)+\rho_{1} \rho_{2} \\
&+\rho_{2}(1-v)+\left(\rho_{1}^{2}-\rho_{1}\right) \nu \\
&+\left[\left(\rho_{1}+\rho_{2}\right) \nu-\rho_{2}\right] \\
& \quad \times \sqrt{\left(\rho_{1}+\rho_{2}+1\right)^{2}-4 \rho_{1}\left(\rho_{2} w+1\right)}
\end{aligned}
$$

$Z_{+}^{*}(w)$

$$
=\frac{1}{2 \rho_{2}}\left[\rho_{1}+\rho_{2}+1+\sqrt{\left(\rho_{1}+\rho_{2}+1\right)^{2}-4 \rho_{2}\left(\rho_{1} w+1\right)}\right] .
$$

(iii) $m=n$ :

$$
\begin{gathered}
p(n, n)=\frac{1-\rho_{1}-\rho_{2}}{2 \pi i} \oint \frac{h(w)}{w^{n+1}} d w, \\
h(w)=\frac{\operatorname{num}(w)}{\operatorname{den}(w)}
\end{gathered}
$$

$\operatorname{num}(w)$

$$
\begin{aligned}
= & -2 \rho_{1} \rho_{2} w+\left(\rho_{1}+\rho_{2}+1\right)\left[\rho_{1} \nu+\rho_{2}(1-\nu)\right] \\
& -\rho_{1} v \sqrt{\left(\rho_{1}+\rho_{2}+1\right)^{2}-4 \rho_{2}\left(\rho_{1} w+1\right)} \\
& -\rho_{2}(1-\nu) \sqrt{\left(\rho_{1}+\rho_{2}+1\right)^{2}-4 \rho_{1}\left(\rho_{2} w+1\right)} .
\end{aligned}
$$

We next evaluate $p(m, n)$ in various asymptotic limits, to gain more insight into the structure of the joint distribution. Writing $p(m, n)=p\left(m, n ; \rho_{1}, \rho_{2}, \nu\right)$ to emphasize the dependence on the model parameters, we clearly have the symmetry relation

$$
p\left(m, n ; \rho_{1}, \rho_{2}, \nu\right)=p\left(n, m ; \rho_{2}, \rho_{1}, 1-\nu\right) .
$$

Thus it is sufficient for the asymptotics to assume that

$$
\rho_{1} \geqslant \rho_{2} \text {, }
$$




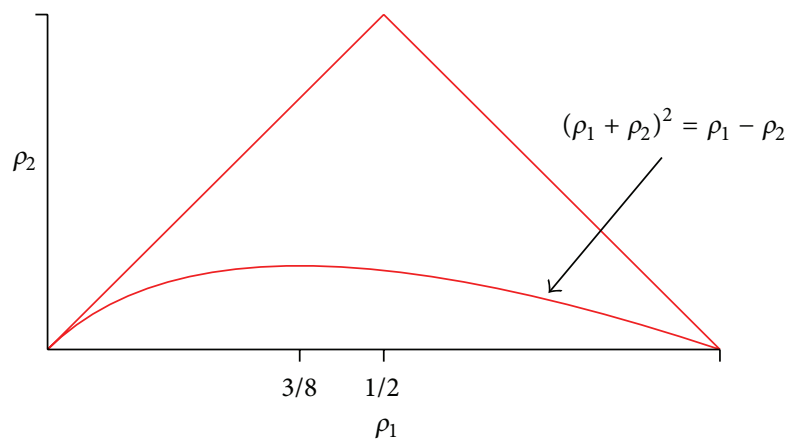

FIGURE 2: A sketch of the parameter domain and the transition curve $\left(\rho_{1}+\rho_{2}\right)^{2}=\rho_{1}-\rho_{2}$.

and we also note that the expressions in Theorem 1 are consistent with (22).

We will show that the asymptotics are quite different whether $\left(\rho_{1}+\rho_{2}\right)^{2}<\rho_{1}-\rho_{2},\left(\rho_{1}+\rho_{2}\right)^{2}>\rho_{1}-\rho_{2}$ or $\left(\rho_{1}+\rho_{2}\right)^{2} \approx$ $\rho_{1}-\rho_{2}$. In Figure 2 we sketch the curve $\left(\rho_{1}+\rho_{2}\right)^{2}=\rho_{1}-\rho_{2}$ in the $\left(\rho_{1}, \rho_{2}\right)$ parameter plane, for $\rho_{1}+\rho_{2}<1$. Note that the curve may also be written as

$$
\rho_{2}=\frac{1}{2}\left[-1-2 \rho_{1}+\sqrt{1+8 \rho_{1}}\right]
$$

and passes through the points $(0,0)$ and $(1,0)$. We first give results for $p(m, n)$ when $\left(\rho_{1}+\rho_{2}\right)^{2}>\rho_{1}-\rho_{2}$, and note that this includes the symmetric case of Flatto [1].

Theorem 2. For $\rho_{1} \geqslant \rho_{2}$ and $\left(\rho_{1}+\rho_{2}\right)^{2}>\rho_{1}-\rho_{2}$ one lets $R=m / n$ be the queue length ratio. The following asymptotic expressions then hold:

(i) $n=O(1), m \rightarrow \infty$

$p(m, n)$

$$
\begin{aligned}
& \sim\left(1-\rho_{1}-\rho_{2}\right) f(0) \frac{m^{n}}{n !}\left(\frac{\rho_{2}}{\sqrt{\left(\rho_{1}+\rho_{2}+1\right)^{2}-4 \rho_{1}}}\right)^{n} \\
& \quad \times\left(\frac{2 \rho_{1}}{\rho_{1}+\rho_{2}+1+\sqrt{\left(\rho_{1}+\rho_{2}+1\right)^{2}-4 \rho_{1}}}\right)^{m} .
\end{aligned}
$$

(ii) $m, n \rightarrow \infty$ with $R=m / n \in\left(R_{c}, \infty\right)$,

$$
\begin{aligned}
& R_{c}=1+\frac{\left[\left(\rho_{1}+\rho_{2}\right)^{2}+\rho_{2}\right]\left[\left(\rho_{1}+\rho_{2}\right)^{2}+\rho_{2}-\rho_{1}\right]}{\rho_{1} \rho_{2}}>1, \\
& p(m, n) \sim \frac{1-\rho_{1}-\rho_{2}}{\sqrt{2 \pi n}} \frac{f_{0}(R)}{\sqrt{M(R)}}\left(\frac{1}{w_{s}}\right)^{n}\left[\frac{1}{Z_{+}\left(w_{s}\right)}\right]^{m-n},
\end{aligned}
$$

where

$$
\begin{aligned}
& w_{s}=w_{s}(R) \\
& =\left(\left(\rho_{1}+\rho_{2}+1\right)^{2} R-4(R+1) \rho_{1}\right. \\
& \left.+\left(\rho_{1}+\rho_{2}+1\right) \sqrt{R^{2}\left(\rho_{1}+\rho_{2}+1\right)^{2}-4 \rho_{1}\left(R^{2}-1\right)}\right) \\
& \quad \times\left(2 \rho_{1} \rho_{2}(R+1)^{2}\right)^{-1}, \\
& Z_{+}\left(w_{s}\right) \\
& =\frac{1}{2 \rho_{1}(R+1)} \\
& \quad \times\left[R\left(\rho_{1}+\rho_{2}+1\right)\right. \\
& \left.\quad+\sqrt{R^{2}\left(\rho_{1}+\rho_{2}+1\right)^{2}-4 \rho_{1}\left(R^{2}-1\right)}\right],
\end{aligned}
$$

$M(R)=\frac{R+1}{R-1}$

$$
\times \frac{\sqrt{R^{2}\left(\rho_{1}+\rho_{2}+1\right)^{2}-4 \rho_{1}\left(R^{2}-1\right)}}{\sqrt{R^{2}\left(\rho_{1}+\rho_{2}+1\right)^{2}-4 \rho_{1}\left(R^{2}-1\right)}-\left(\rho_{1}+\rho_{2}+1\right)}
$$

and $f_{0}(R)=f\left(w_{s}\right)=f\left(w_{s}(R)\right)$ can be computed from (12)-(14).

(iii) $m, n \rightarrow \infty$ with $R=R_{c}+\xi / \sqrt{n}$ (thus $R-R_{c}=$ $\left.O\left(n^{-1 / 2}\right)\right)$

$$
\begin{aligned}
& p(m, n) \sim C_{+}\left(\rho_{1}+\rho_{2}\right)^{2 n}\left[\frac{\rho_{1}\left(\rho_{1}+\rho_{2}\right)}{\left(\rho_{1}+\rho_{2}\right)^{2}+\rho_{2}}\right]^{m-n} \\
& \times \frac{1}{\sqrt{2 \pi}} \int_{\xi_{1}}^{\infty} e^{-u^{2} / 2} d u \\
& \xi_{1}=\xi \frac{1}{R_{c}-1}\left[\frac{R_{c}}{R_{c}-1}+\frac{2 \rho_{1} \rho_{2}}{\left(\left(\rho_{1}+\rho_{2}\right)^{2}+\rho_{2}-\rho_{1}\right)^{2}}\right]^{-1 / 2}, \\
& C_{+}=\left(1-\rho_{1}-\rho_{2}\right) \\
& \times \frac{\left(\rho_{1}+\rho_{2}\right)^{4}-\left(\rho_{1}-\rho_{2}\right)^{2}}{\rho_{1}\left(\rho_{1}+\rho_{2}\right)^{2}} \\
& \times \frac{\left(\rho_{1}+\rho_{2}\right)^{2}(1-\nu)+\rho_{1}}{\left(\rho_{1}+\rho_{2}\right)^{2}+\rho_{2} \nu+\rho_{1}(1-\nu)} .
\end{aligned}
$$

(iv) $m, n \rightarrow \infty$ with $R=m / n \in\left(1, R_{c}\right)$

$$
p(m, n) \sim C_{+}\left(\rho_{1}+\rho_{2}\right)^{2 n}\left[\frac{\rho_{1}\left(\rho_{1}+\rho_{2}\right)}{\left(\rho_{1}+\rho_{2}\right)^{2}+\rho_{2}}\right]^{m-n} .
$$


(v) $m=n \rightarrow \infty$

$$
\begin{gathered}
p(n, n) \sim C_{0}\left(\rho_{1}+\rho_{2}\right)^{2 n}, \\
C_{0}=\frac{1-\rho_{1}-\rho_{2}}{\left(\rho_{1}+\rho_{2}\right)^{2}} \frac{\left(\rho_{1}+\rho_{2}\right)^{4}-\left(\rho_{1}-\rho_{2}\right)^{2}}{\left(\rho_{1}+\rho_{2}\right)^{2}+\rho_{2} \nu+\rho_{1}(1-\nu)} .
\end{gathered}
$$

(vi) $m, n \rightarrow \infty$ with $R=m / n \in\left(R_{c}^{*}, 1\right)$,

$$
\begin{gathered}
R_{c}^{*} \equiv \frac{\rho_{1} \rho_{2}}{\rho_{1} \rho_{2}+\left[\left(\rho_{1}+\rho_{2}\right)^{2}+\rho_{1}-\rho_{2}\right]\left[\left(\rho_{1}+\rho_{2}\right)^{2}+\rho_{1}\right.} \\
p(m, n) \sim C_{-}\left(\rho_{1}+\rho_{2}\right)^{2 m}\left[\frac{\rho_{2}\left(\rho_{1}+\rho_{2}\right)}{\left(\rho_{1}+\rho_{2}\right)^{2}+\rho_{1}}\right]^{n-m}, \\
C_{-}=\left(1-\rho_{1}-\rho_{2}\right) \frac{\left(\rho_{1}+\rho_{2}\right)^{4}-\left(\rho_{1}-\rho_{2}\right)^{2}}{\rho_{2}\left(\rho_{1}+\rho_{2}\right)^{2}} \\
\times \frac{\rho_{2}+v\left(\rho_{1}+\rho_{2}\right)^{2}}{\left(\rho_{1}+\rho_{2}\right)^{2}+\rho_{2} v+\rho_{1}(1-v)} .
\end{gathered}
$$

(vii) $m, n \rightarrow \infty$ with $R=R_{c}^{*}+\eta / \sqrt{n}$ (thus $R-R_{c}^{*}=$ $\left.O\left(n^{-1 / 2}\right)\right)$

$$
\begin{aligned}
& p(m, n) \sim C_{-}\left(\rho_{1}+\rho_{2}\right)^{2 m} \\
& \times\left[\frac{\rho_{2}\left(\rho_{1}+\rho_{2}\right)}{\left(\rho_{1}+\rho_{2}\right)^{2}+\rho_{1}}\right]^{n-m} \frac{1}{\sqrt{2 \pi}} \int_{-\infty}^{\eta_{1}} e^{-u^{2} / 2} d u, \\
& \eta_{1}=\eta \frac{1}{1-R_{c}^{*}}\left[\frac{R_{c}^{*}}{1-R_{c}^{*}}+\frac{2 \rho_{1} \rho_{2} R_{c}^{*}}{\left(\left(\rho_{1}+\rho_{2}\right)^{2}+\rho_{1}-\rho_{2}\right)^{2}}\right]^{-1 / 2} .
\end{aligned}
$$

(viii) $m, n \rightarrow \infty$ with $R=m / n \in\left(0, R_{c}^{*}\right)$

$$
\begin{aligned}
& p(m, n) \sim \frac{1-\rho_{1}-\rho_{2}}{\sqrt{2 \pi n}} \frac{g_{0}(R)}{\sqrt{M_{*}(R)}}\left(\frac{1}{w_{s}^{*}}\right)^{m}\left[\frac{1}{Z_{+}^{*}\left(w_{s}^{*}\right)}\right]^{n-m}, \\
& w_{s}^{*}=w_{s}^{*}(R) \\
&=\left(R \left[\left(\rho_{1}+\rho_{2}+1\right)^{2}-4 \rho_{2}(R+1)\right.\right. \\
&\left.\left.\quad+\left(\rho_{1}+\rho_{2}+1\right) \sqrt{\left(\rho_{1}+\rho_{2}+1\right)^{2}-4 \rho_{2}\left(1-R^{2}\right)}\right]\right) \\
& \times\left(2 \rho_{1} \rho_{2}(R+1)^{2}\right)^{-1},
\end{aligned}
$$

$$
\begin{aligned}
Z_{+}^{*}\left(w_{s}^{*}\right)= & \frac{1}{2 \rho_{2}(R+1)} \\
& \times\left[1+\rho_{1}+\rho_{2}+\sqrt{\left(1+\rho_{1}+\rho_{2}\right)^{2}-4 \rho_{2}\left(1-R^{2}\right)}\right],
\end{aligned}
$$

$M_{*}(R)$

$$
=R \frac{1+R}{1-R}
$$

$$
\times \frac{\sqrt{\left(\rho_{1}+\rho_{2}+1\right)^{2}-4 \rho_{2}\left(1-R^{2}\right)}}{\sqrt{\left(\rho_{1}+\rho_{2}+1\right)^{2}-4 \rho_{2}\left(1-R^{2}\right)}-R\left(\rho_{1}+\rho_{2}+1\right)}
$$

and $g_{0}(R)=g\left(w_{s}^{*}\right)=g\left(w_{s}^{*}(R)\right)$ can be computed from (14), (16), and (17).

(ix) $m=O(1), n \rightarrow \infty$

$p(m, n)$

$$
\begin{aligned}
& \sim\left(1-\rho_{1}-\rho_{2}\right) g(0) \frac{n^{m}}{m !}\left(\frac{\rho_{1}}{\sqrt{\left(\rho_{1}+\rho_{2}+1\right)^{2}-4 \rho_{2}}}\right)^{m} \\
& \quad \times\left(\frac{2 \rho_{2}}{1+\rho_{1}+\rho_{2}+\sqrt{\left(1+\rho_{1}+\rho_{2}\right)^{2}-4 \rho_{2}}}\right)^{n} .
\end{aligned}
$$

Note that, in view of (12) and (16),

$f(0)$

$$
\begin{aligned}
& =\left(\rho_{2}^{2}(1-v)+\rho_{1} \rho_{2}-\rho_{2}(1-v)+\left(\rho_{1}^{2}+\rho_{1}\right) \nu\right. \\
& \left.\quad+\left[\rho_{2}-\left(\rho_{1}+\rho_{2}\right) v\right] \sqrt{\left(\rho_{1}+\rho_{2}+1\right)^{2}-4 \rho_{2}}\right) \\
& \times\left(\left(\rho_{1}+\rho_{2}+1\right)\left[\rho_{1} v+\rho_{2}(1-v)\right]\right. \\
& \quad-\rho_{1} v \sqrt{\left(\rho_{1}+\rho_{2}+1\right)^{2}-4 \rho_{2}} \\
& \left.-\rho_{2}(1-v) \sqrt{\left(\rho_{1}+\rho_{2}+1\right)^{2}-4 \rho_{1}}\right)^{-1},
\end{aligned}
$$


$g(0)$

$$
\begin{aligned}
& =\left(\rho_{2}^{2}(1-v)+\rho_{1} \rho_{2}+\rho_{2}(1-v)+\left(\rho_{1}^{2}-\rho_{1}\right) \nu\right. \\
& \left.+\left[\left(\rho_{1}+\rho_{2}\right) \nu-\rho_{2}\right] \sqrt{\left(\rho_{1}+\rho_{2}+1\right)^{2}-4 \rho_{2}}\right) \\
& \times\left(\left(\rho_{1}+\rho_{2}+1\right)\left[\rho_{1} \nu+\rho_{2}(1-v)\right]\right. \\
& -\rho_{1} v \sqrt{\left(\rho_{1}+\rho_{2}+1\right)^{2}-4 \rho_{2}} \\
& \left.-\rho_{2}(1-v) \sqrt{\left(\rho_{1}+\rho_{2}+1\right)^{2}-4 \rho_{1}}\right)^{-1} .
\end{aligned}
$$

In Theorem 2 we listed the expansions of $p(m, n)$ in order of decreasing $R$, from $R=\infty$ in (25) to $R=0$ in (46). In the symmetric case $\rho_{1}=\rho_{2}$ and $v=1 / 2$, and then $p(m, n)=p(n, m)$ and Theorem 2 is consistent with the results of Flatto in [1] (there the cases $m=O(1), n \rightarrow$ $\infty$ and $n=O(1), m \rightarrow \infty$ were not considered, and the limits where $R \approx R_{c}, R_{c}^{*}$ were not treated in as much detail). Note that (34) and (38) correspond to "product form" approximations to $p(m, n)$. The expressions in (25) and (46) are actually exact when $n=0$ and $m=0$, respectively, in view of our comments below (9). Our analysis in Section 4 will also indicate how to compute higher order correction terms in the various asymptotic series.

Next we take $\left(\rho_{1}+\rho_{2}\right)^{2}<\rho_{1}-\rho_{2}$. Now some of the asymptotic results for $p(m, n)$ will be very different, while in other ranges of $R=m / n$ they will be similar to those in Theorem 2. We emphasize below the formulas that are different.

Theorem 3. For $\rho_{1}>\rho_{2}$ and $\left(\rho_{1}+\rho_{2}\right)^{2}<\rho_{1}-\rho_{2}$ one has the following asymptotic expansions:

(i) $m, n \rightarrow \infty$ with $m>n$ and $m-n=O(1)$

$$
\begin{gathered}
p(m, n) \sim \bar{C}_{+} \frac{\sqrt{w_{b}}}{\sqrt{\pi}} n^{-3 / 2} w_{b}^{-n}\left(\frac{2 \rho_{1}}{\rho_{1}+\rho_{2}+1}\right)^{m-n} \\
\times\left[\frac{2 \sqrt{\rho_{1} \rho_{2}}}{\rho_{1}+\rho_{2}+1}(m-n)+\Delta_{+}\right], \\
w_{b}=\frac{\left(\rho_{1}+\rho_{2}+1\right)^{2}-4 \rho_{1}}{4 \rho_{1} \rho_{2}}, \\
\bar{C}_{+}=\frac{1-\rho_{1}-\rho_{2}}{2} \frac{1-\sqrt{\rho_{1}-\rho_{2}}}{\sqrt{\rho_{1}-\rho_{2}}-\left(\rho_{1}+\rho_{2}\right)} \\
\times \frac{\sqrt{\rho_{1}-\rho_{2}}+1 / 2-(\nu-1 / 2)\left(\rho_{1}+\rho_{2}\right)}{(1 / 2)\left(\rho_{1}+\rho_{2}+1\right)-(\nu-1) \sqrt{\rho_{1}-\rho_{2}}},
\end{gathered}
$$

$$
\begin{aligned}
\Delta_{+}=(2 & \sqrt{\rho_{1} \rho_{2}}\left[\rho_{2}(1-v)-\rho_{1}\right. \\
& \left.\left.+\frac{1}{4}\left(\rho_{1}+\rho_{2}+1\right)^{2}\right]\right) \\
\times & \left(\left[\sqrt{\rho_{1}-\rho_{2}}-\left(\rho_{1}+\rho_{2}\right)\right]\right. \\
\times & {\left[1-\sqrt{\rho_{1}-\rho_{2}}\right] } \\
\times & {\left.\left[\frac{1}{2}\left(\rho_{1}+\rho_{2}+1\right)-(\nu-1) \sqrt{\rho_{1}-\rho_{2}}\right]\right)^{-1} . }
\end{aligned}
$$

(ii) $m=n \rightarrow \infty$

$$
\begin{gathered}
p(n, n) \sim \bar{C} \frac{\sqrt{w_{b}}}{\sqrt{\pi}} n^{-3 / 2} w_{b}^{-n}, \\
\bar{C}=\left(\left(1-\rho_{1}-\rho_{2}\right) w_{b}\left(\rho_{1} \rho_{2}\right)^{3 / 2}\right. \\
\left.\times\left[\frac{1}{2}+\sqrt{\rho_{1}-\rho_{2}}-\left(\nu-\frac{1}{2}\right)\left(\rho_{1}+\rho_{2}\right)\right]\right) \\
\times\left(\left[\sqrt{\rho_{1}-\rho_{2}}-\left(\rho_{1}+\rho_{2}\right)\right]^{2}\right. \\
\left.\times\left[\frac{1}{2}\left(\rho_{1}+\rho_{2}+1\right)-(\nu-1) \sqrt{\rho_{1}-\rho_{2}}\right]^{2}\right)^{-1} .
\end{gathered}
$$

(iii) $m, n \rightarrow \infty$ with $R=m / n \in\left(R_{d}, 1\right)$ (including the limit $n-m=O(1)$ ),

$$
\begin{gathered}
R_{d}=\frac{\left(\rho_{1}+\rho_{2}+1\right)^{2}-4 \rho_{1}}{\left(\rho_{1}+\rho_{2}+1+2 \sqrt{\rho_{1}-\rho_{2}}\right)^{2}-4 \rho_{2}}<1, \\
p(m, n) \sim \bar{C}_{-}\left(R-R_{d}\right)^{-3 / 2} \frac{\sqrt{w_{b}}}{\sqrt{\pi}} n^{-3 / 2} w_{b}^{-m} \\
\times\left(\frac{2 \rho_{2}}{\rho_{1}+\rho_{2}+1+2 \sqrt{\rho_{1}-\rho_{2}}}\right)^{n-m},
\end{gathered}
$$

$\bar{C}_{-}$

$$
\begin{aligned}
& =\left(\rho_{1}-\rho_{2}\right)^{3 / 4}\left(1-\rho_{1}-\rho_{2}\right) \sqrt{\rho_{1} \rho_{2}} \\
& \times\left[\frac{\rho_{1}+\rho_{2}+1+2 \sqrt{\rho_{1}-\rho_{2}}}{(1 / 4)\left(\rho_{1}+\rho_{2}+1+2 \sqrt{\rho_{1}-\rho_{2}}\right)^{2}-\rho_{2}}\right]^{3 / 2} \\
& \times\left(\left[\frac{1}{2}+\sqrt{\rho_{1}-\rho_{2}}+\left(\rho_{1}+\rho_{2}\right)\left(\frac{1}{2}-\nu\right)\right]\right. \\
& \left.\quad \times\left[\frac{1}{4}\left(\rho_{1}+\rho_{2}+1\right)^{2}-\rho_{1}(1-v)\right]\right) \\
& \times\left(\left[\sqrt{\rho_{1}-\rho_{2}}-\left(\rho_{1}+\rho_{2}\right)\right]^{2}\right. \\
& \left.\quad \times\left[\frac{1}{2}\left(\rho_{1}+\rho_{2}+1\right)+\sqrt{\rho_{1}-\rho_{2}}(1-\nu)\right]^{2}\right)^{-1} .
\end{aligned}
$$


(iv) $m, n \rightarrow \infty$ with $R=R_{d}+S / \sqrt{n}$ (thus $R-R_{d}=$ $\left.O\left(n^{-1 / 2}\right)\right)$

$$
\begin{aligned}
& p(m, n)=\frac{1-\rho_{1}-\rho_{2}}{\sqrt{2 \pi n}} w_{b}^{-m}\left[Z_{+}^{*}\left(w_{b}\right)\right]^{m-n} \\
& \times\left[\frac{g\left(w_{b}\right)}{\sqrt{M_{d}}} e^{-S_{1}^{2} / 2}-\frac{K \sqrt{w_{b}}}{M_{d}^{3 / 4}} e^{-S_{1}^{2} / 4}\right. \\
& \left.\times \frac{D_{1 / 2}\left(-S_{1}\right)}{n^{1 / 4}}+O\left(n^{-1 / 2}\right)\right], \\
& M_{d}=\frac{R_{d}}{1-R_{d}}+\frac{R_{d} \rho_{1} \rho_{2} w_{b}}{2\left(\rho_{1}-\rho_{2}\right)}, \\
& S_{1}=\frac{1}{1-R_{d}} \frac{1}{\sqrt{M_{d}}} S, \\
& K=\left(2 \sqrt{\rho_{1} \rho_{2}}\left[\frac{1}{2}+\sqrt{\rho_{1}-\rho_{2}}+\left(\rho_{1}+\rho_{2}\right)\left(\frac{1}{2}-v\right)\right]\right. \\
& \left.\times\left[\frac{1}{4}\left(\rho_{1}+\rho_{2}+1\right)^{2}-\rho_{1}(1-\nu)\right]\right) \\
& \times\left(\left[\sqrt{\rho_{1}-\rho_{2}}-\left(\rho_{1}+\rho_{2}\right)\right]^{2}\right. \\
& \left.\times\left[\frac{1}{2}\left(\rho_{1}+\rho_{2}+1\right)+\sqrt{\rho_{1}-\rho_{2}}(1-\nu)\right]^{2}\right)^{-1},
\end{aligned}
$$

and $D_{1 / 2}(\cdot)$ is the parabolic cylinder function of order $1 / 2$.

(v) For $n=O(1)$ and $m \rightarrow \infty$, (25) applies. For $m, n \rightarrow$ $\infty$ with $R=m / n \in(1, \infty)$, (27)-(30) hold. For $m, n \rightarrow \infty$ with $R=m / n \in\left(0, R_{d}\right)$, (42)-(45) hold. For $m=O(1)$ and $n \rightarrow \infty$, (46) applies.

Thus when $\left(\rho_{1}+\rho_{2}\right)^{2}<\rho_{1}-\rho_{2}$ the asymptotics of $p(m, n)$ are different in eight ranges of $R=m / n$, but the final result in four of these is the same as the case $\left(\rho_{1}+\rho_{2}\right)^{2}>\rho_{1}-\rho_{2}$. Note however that if $\left(\rho_{1}+\rho_{2}\right)^{2}<\rho_{1}-\rho_{2}(27)$ applies for all $R>1$, so the transition for $R \approx R_{c}$ is now absent. Also, if $\left(\rho_{1}+\rho_{2}\right)^{2}<$ $\rho_{1}-\rho_{2},(42)$ applies for $R \in\left(0, R_{d}\right)$ rather than $R \in\left(0, R_{c}^{*}\right)$. In (57), $g\left(w_{b}\right)$ is computed by setting $w=w_{b}$, cf. (49), in (16), and we also note that $2 \rho_{2} Z_{+}^{*}\left(w_{b}\right)=\rho_{1}+\rho_{2}+1+2 \sqrt{\rho_{1}-\rho_{2}}$.

The results in (49)-(60) are very different in form from the symmetric case asymptotics in [1]. For example, the diagonal probabilities in (52) contain the additional algebraic factor $n^{-3 / 2}$. In (57) the parabolic cylinder function can be computed, for example, from

$$
D_{p}(Z)=\frac{e^{Z^{2} / 4}}{i \sqrt{2 \pi}} \int_{\mathrm{Br}} e^{-Z u} e^{u^{2} / 2} u^{p} d u,
$$

where $\mathrm{Br}$ is a vertical contour in the complex $u$-plane with $\operatorname{Re}(u)>0$, and we have $p=1 / 2$ for the correction term in (57). Also, $D_{0}(Z)=e^{-Z^{2} / 4}$ and for $p=N=0,1,2,3, \ldots$ we can express $D_{N}(Z)$ in terms of a Hermite polynomial. In (57) we give a two-term asymptotic approximation in the transition range $R \approx R_{d}$. Also, it may be shown that the leading term in (57) is just the limiting form of (42), as $R \rightarrow$ $R_{d}$. The expression in (55) applies both to $m, n \rightarrow \infty$ with $R_{d}<R<1$, and to $m, n \rightarrow \infty$ with $n-m=O(1)$. In the latter case we can approximate the factor $\left(R-R_{d}\right)^{-3 / 2}$ by $\left(1-R_{d}\right)^{-3 / 2}$. The parabolic cylinder function $D_{1 / 2}\left(-S_{1}\right)$ has the asymptotic behaviors

$$
\begin{gathered}
D_{1 / 2}\left(-S_{1}\right) \sim e^{-S_{1}^{2} / 4} \sqrt{-S_{1}}, \quad S_{1} \longrightarrow-\infty, \\
D_{1 / 2}\left(-S_{1}\right) \sim e^{S_{1}^{2} / 4} S_{1}^{-3 / 2} \frac{-1}{\sqrt{2}}, \quad S_{1} \longrightarrow+\infty,
\end{gathered}
$$

so as $S_{1} \rightarrow+\infty$ in (57) (corresponding to $R>R_{d}$ ) the $O\left(n^{-1 / 4}\right)$ correction term begins to dominate the leading term (which occurs for $e^{-S_{1}^{2} / 2} \ll n^{-1 / 4}$ or $\left|S_{1}\right| \gg \sqrt{(1 / 2) \log n}$ ). Then we can show that the expression in (57) for $S_{1} \rightarrow+\infty$ agrees with (55) for $R \downarrow R_{d}$, and hence the two expansions asymptotically match. Note also that the scaling in (57) has $m=n R_{d}+O(\sqrt{n})$.

Next we study the transition range $\left(\rho_{1}+\rho_{2}\right)^{2} \approx \rho_{1}-\rho_{2}$ in parameter space. This will lead to a new set of asymptotic results which will show, for example, how the formula in (52) for $p(n, n)$ changes to the purely geometric approximation in (35). To quantify the closeness to the curve $\left(\rho_{1}+\rho_{2}\right)^{2}=\rho_{1}-\rho_{2}$ in Figure 2 we write

$$
\left(\rho_{1}+\rho_{2}\right)^{2}-\left(\rho_{1}-\rho_{2}\right)=\delta \beta
$$

where $\delta \rightarrow 0^{+}$and $\beta$ can have either sign. If $\beta=0$ we are exactly on the transition curve. For small $\delta$, in certain ranges of $R=m / n$, the results in Theorems 2 and 3 still apply. For example, if $m=O(1)$ and $n \rightarrow \infty$ or $n=O(1)$ and $m \rightarrow \infty$ Theorems 2 and 3 agree, and then no transition range result is needed. As $\left(\rho_{1}+\rho_{2}\right)^{2} \downarrow \rho_{1}-\rho_{2}, R_{c} \downarrow 1$ in (26), and then (27) will hold for all $R \in(1, \infty)$ in the transition case. Thus for $R=m / n>1$ the transition case will require a new asymptotic result only if $R \approx 1$. For $R<1$ the asymptotic result in (42) will apply for $R<R_{c}^{*}$, where now, since $\left(\rho_{1}+\rho_{2}\right)^{2} \approx \rho_{1}-\rho_{2}$, $R_{c}^{*} \approx R_{d}$ (cf. (37) and (54)). Then if $R<1$, we will need different asymptotic results only when $R \in\left[R_{d}^{0}, 1\right]$, including $R \approx R_{d}^{0}$ and $R \approx 1$, where

$$
R_{d}^{0}=\frac{1-a^{2}}{1+4 a+11 a^{2}}, \quad a=\rho_{1}+\rho_{2}
$$


Note that $R_{d}^{0}$ is the limit of both $R_{d}$ and $R_{c}^{*}$, if we replace $\rho_{1}-\rho_{2}$ by $\left(\rho_{1}+\rho_{2}\right)^{2}=a^{2}$, as (37) leads to (setting $a=\rho_{1}+\rho_{2}, b=$ $\left.\sqrt{\rho_{1}-\rho_{2}}\right)$

$$
\begin{aligned}
R_{c}^{*} & =\frac{\rho_{1} \rho_{2}}{\rho_{1} \rho_{2}+\left[\left(\rho_{1}+\rho_{2}\right)^{2}+\rho_{1}-\rho_{2}\right]\left[\left(\rho_{1}+\rho_{2}\right)^{2}+\rho_{1}\right]} \\
& =\frac{(1 / 4)\left(a^{2}-b^{4}\right)}{(1 / 4)\left(a^{2}-b^{4}\right)+\left(a^{2}+b^{2}\right)\left[a^{2}+(1 / 2)\left(a+b^{2}\right)\right]} \\
& \longrightarrow \frac{(1 / 4) a^{2}\left(1-a^{2}\right)}{(1 / 4) a^{2}\left(1-a^{2}\right)+a^{2}\left(3 a^{2}+a\right)} \\
& =\frac{1-a^{2}}{1+4 a+11 a^{2}},
\end{aligned}
$$

since $\delta \rightarrow 0^{+}$corresponds to $b \rightarrow a$. Similarly, (54) leads to $R_{d} \rightarrow R_{d}^{0}$ in this limit.

Since we will now have the state variables $m$ and $n$ large, and also $\delta$ small, it is necessary to relate these. In Theorem 4 we summarize the transition case results, scaling $m$ and $n$ in terms of $\delta$.

Theorem 4. For $\rho_{1}>\rho_{2}$ and $\left(\rho_{1}+\rho_{2}\right)^{2}-\left(\rho_{1}-\rho_{2}\right)=\beta \delta$ with $\delta \rightarrow 0^{+}$, one has the following, where $a=\rho_{1}+\rho_{2}$ and $b=$ $\sqrt{\rho_{1}-\rho_{2}}$.

(i) $m=n \rightarrow \infty, n=y / \delta^{2}=O\left(\delta^{-2}\right)$

$$
\begin{gathered}
p(n, n) \sim \frac{1}{\sqrt{\pi n}} \frac{2(1-a) \sqrt{1-a^{2}}}{3 a+1-2 a v} w_{b}^{-n} \\
\times\left[1+2 \beta_{1} \sqrt{y} e^{\beta_{1}^{2} y} \int_{-\infty}^{\beta_{1} \sqrt{y}} e^{-u^{2}} d u\right], \\
\beta=a \sqrt{1-a^{2}} \beta_{1} .
\end{gathered}
$$

(ii) $n=y / \delta^{2}=O\left(\delta^{-2}\right), m-n=z / \delta=O\left(\delta^{-1}\right), m>n$

$$
\begin{aligned}
p(m, n) \sim 2 & (1-a) \sqrt{\frac{1-a}{1+a}} \frac{1}{\sqrt{\pi n}} w_{b}^{-n}\left(\frac{2 \rho_{1}}{\rho_{1}+\rho_{2}+1}\right)^{m-n} \\
& \times\left[e^{-\tilde{z}^{2} / 4}+2 \beta_{1} \sqrt{y} e^{\beta_{1}^{2} y} e^{-\tilde{z} \beta_{1} \sqrt{y}} \int_{-\infty}^{\beta_{1} \sqrt{y}-\tilde{z} / 2} e^{-u^{2}} d u\right], \\
\widetilde{z} & =\sqrt{\frac{1-a}{1+a}} \frac{z}{\sqrt{y}}=\sqrt{\frac{1-a}{1+a}} \frac{m-n}{\sqrt{n}}=O(1)
\end{aligned}
$$

(iii) $n=y / \delta^{2}=O\left(\delta^{-2}\right), R=m / n \in\left(R_{d}^{0}, 1\right], m<n$

$$
\begin{aligned}
p(m, n) \sim & 2(1-a) \sqrt{\frac{1-a}{1+a}} \frac{1}{\sqrt{\pi n}} \frac{1-a+2 v a}{3 a+1-2 v a} \sqrt{\frac{1-R_{d}^{0}}{R-R_{d}^{0}}} \\
& \times w_{b}^{-m}\left[Z_{+}^{*}\left(w_{b}\right)\right]^{m-n} \\
& \times\left[1+2 \beta_{2} \sqrt{y} e^{\beta_{2}^{2}} \int_{-\infty}^{\beta_{2} \sqrt{y}} e^{-u^{2}} d u\right],
\end{aligned}
$$

$$
\beta=a \sqrt{1-a^{2}} \sqrt{\frac{1-R_{d}^{0}}{R-R_{d}^{0}}} \beta_{2} .
$$

(iv) $m, n \rightarrow \infty, R=m / n=R_{d}^{0}+S^{0} / \sqrt{n}$ (thus $R-R_{d}^{0}=$ $\left.O\left(n^{-1 / 2}\right)\right)$

$$
\begin{aligned}
p(m, n) \sim & \sqrt{\frac{2\left(1-a^{2}\right)}{\pi}} \frac{1-a+2 v a}{1+3 a-2 v a} w_{b}^{-m}\left[Z_{+}^{*}\left(w_{b}\right)\right]^{m-n} \\
& \times\left[R_{d}^{0}\left(\frac{1}{1-R_{d}^{0}}+\frac{1-a^{2}}{8 a^{2}}\right)\right]^{-1 / 4} n^{-1 / 4} e^{-\left(S_{1}^{0}\right)^{2} / 4} \\
& \times D_{-1 / 2}\left(-S_{1}^{0}\right), \\
S_{1}^{0}= & \frac{1}{\sqrt{R_{d}^{0}}\left(1-R_{d}^{0}\right)}\left[\frac{1}{1-R_{d}^{0}}+\frac{1-a^{2}}{8 a^{2}}\right]^{-1 / 2} S^{0},
\end{aligned}
$$

and $D_{-1 / 2}(\cdot)$ is the parabolic cylinder function of order $-1 / 2($ see $(61))$.

(v) $m, n \rightarrow \infty, n=y_{1} / \delta^{4}=O\left(\delta^{-4}\right), R=R_{d}^{0}+S^{0} / \sqrt{n}=$ $R_{d}^{0}+O\left(\delta^{2}\right)$

$$
\begin{aligned}
p(m, n) \sim & \frac{2 \sqrt{1-a^{2}}}{n^{1 / 4}} \frac{1-a+2 v a}{1+3 a-2 v a}\left[R_{d}^{0}\left(\frac{1}{1-R_{d}^{0}}+\frac{1-a^{2}}{8 a^{2}}\right)\right]^{-1 / 4} \\
& \times w_{b}^{-m}\left[Z_{+}^{*}\left(w_{b}\right)\right]^{m-n} \mathscr{L}\left(S_{1}^{0} ; y_{1}^{1 / 4} \beta_{3}\right),
\end{aligned}
$$

$$
\beta_{3}=\beta a \sqrt{1-a^{2}}\left(R_{d}^{0}\right)^{-1 / 4}\left[\frac{1}{1-R_{d}^{0}}+\frac{1-a^{2}}{8 a^{2}}\right]^{-1 / 4}
$$

and $\mathscr{L}$ is defined by the contour integral

$$
\mathscr{L}(Z ; \gamma)=\frac{1}{2 \pi i} \int_{\mathrm{Br}_{+}} \frac{e^{Z u} e^{u^{2} / 2}}{-\gamma+\sqrt{u}} d u,
$$

where $\mathrm{Br}_{+}$is to the right of all singularities of the integrand, including the pole at $u=\gamma^{2}$ if $\gamma>0$.

If $\left(\rho_{1}+\rho_{2}\right)^{2}=\rho_{1}-\rho_{2}$ then $\beta=\beta_{1}=0$ and the term with the integral in (66) is absent. In Theorem 4 we wrote most 
results in terms of $a=\rho_{1}+\rho_{2}$ and $\beta$. As $\beta$ (or $\beta_{1}$ ) $\rightarrow+\infty$ it can be shown that (66) asymptotically matches to (35), and as $\beta \rightarrow-\infty$, (66) matches to (52). Similarly as $\beta \rightarrow \infty$ (68) matches to (27) while as $\beta \rightarrow-\infty$ (68) matches to (48). Some of these asymptotic matching relations are further discussed in Section 4. In (70), $\beta_{2}$ depends on $\beta$ and also on the ratio $R=m / n$, in view of (71). The results in Theorem 4 show that for $\delta \rightarrow 0^{+}$it is natural to scale $n=O\left(\delta^{-2}\right)$, and then either $m=O\left(\delta^{-2}\right)$ independently, or $m-n=$ $O\left(\delta^{-1}\right)$. The expression in (74) is similar to, but somewhat more complicated than, that in (72). The function $\mathscr{L}$ in (76) reduces to $(2 \pi)^{-1 / 2} D_{-1 / 2}(Z) e^{-Z^{2} / 4}$ in the limit $\gamma \rightarrow 0$, and thus (72) may be viewed as a special case of (74)-(76). Note also that the results in Theorem 4 can be rewritten without introducing $\beta$ and $\delta$ in (63), as for example

$$
\begin{aligned}
\beta_{1} \sqrt{y}= & \frac{1}{a \sqrt{1-a^{2}}} \\
& \times\left[\left(\rho_{1}+\rho_{2}\right)^{2}-\left(\rho_{1}-\rho_{2}\right)\right] \sqrt{n}, \quad a=\rho_{1}+\rho_{2},
\end{aligned}
$$

and $\beta_{3} y_{1}^{1 / 4}$ is proportional to $\beta \delta n^{1 / 4}$, and $\beta \delta$ may be written in terms of the original parameters $\rho_{1}, \rho_{2}$.

Next we consider some different asymptotic limits, those of "light" and "heavy" traffic. Light traffic corresponds to infrequent arrivals, where $\rho_{1}, \rho_{2} \rightarrow 0$. Heavy traffic corresponds to nearly unstable systems, where $\rho_{1}+\rho_{2} \uparrow 1$. It turns out that the present model has two possible heavy traffic limits. In the first, which we call HTL1, we have $\rho_{1}+\rho_{2} \uparrow 1$ with a fixed $0<\rho_{2}<1$. Then most of the probability mass will occur in the range where $m$ and $n$ are large, but with $m-n=O(1)$. More precisely, if $\varepsilon=1-\rho_{1}-\rho_{2} \rightarrow 0^{+}$then $m$ and $n$ must be scaled to be $O\left(\varepsilon^{-1}\right)$, but with the difference $m-n$ fixed. In the second heavy traffic limit (HTL2) we again set $\varepsilon=1-\rho_{1}-\rho_{2} \rightarrow 0^{+}$but now let $\rho_{2} \rightarrow 0^{+}$, with $\rho_{2}=O(\varepsilon)$. Now the probability mass will become more spread out, with appreciable mass anywhere in the range $X>Y>0$, where $m=X / \varepsilon$ and $n=Y / \varepsilon$. The light and heavy traffic results are summarized below as Theorem 5 .

Theorem 5. (i) Light traffic: For $\rho_{1}, \rho_{2} \rightarrow 0$ one has

$$
p(m, n) \sim \rho_{1}^{m} \rho_{2}^{n}\left(\begin{array}{c}
m+n \\
n
\end{array}\right) .
$$

(ii) Heavy traffic limit 1: $\varepsilon=1-\rho_{1}-\rho_{2} \rightarrow 0^{+}$with $0<$ $\rho_{2}<1, n=Y / \varepsilon=O\left(\varepsilon^{-1}\right), m-n=\ell=O(1)$

$$
\begin{gathered}
p(m, n) \sim \varepsilon \frac{4 \rho_{2}\left(2-v-\rho_{2}\right)}{2-\rho_{2}+\left(2 \rho_{2}-1\right) \nu}\left(\frac{1-\rho_{2}}{1+\rho_{2}}\right)^{\ell} e^{-2 Y}, \quad \ell \geqslant 1, \\
p(n, n) \sim \varepsilon \frac{4 \rho_{2}\left(1-\rho_{2}\right)}{2-\rho_{2}+\left(2 \rho_{2}-1\right) \nu} e^{-2 Y}, \quad \ell=0, \quad(80) \\
p(m, n) \sim \varepsilon \frac{4\left(1-\rho_{2}\right)\left(\rho_{2}+\nu\right)}{2-\rho_{2}+\left(2 \rho_{2}-1\right) \nu}\left(\frac{\rho_{2}}{2-\rho_{2}}\right)^{-\ell} e^{-2 Y}, \quad \ell \leqslant-1 .
\end{gathered}
$$

(iii) Heavy traffic limit 2: $\varepsilon=1-\rho_{1}-\rho_{2} \rightarrow 0^{+}$with $\rho_{2}=\gamma \varepsilon=O(\varepsilon), n=Y / \varepsilon, m=X / \varepsilon$

$$
\begin{aligned}
& p(m, n) \\
& \sim \frac{\varepsilon^{2}}{2 \pi i} \int_{\mathrm{Br}_{+}} f_{0}(\xi) e^{Y \xi} \\
& \times \exp \left\{\frac{Y-X}{2}\left[2 \gamma+1+\sqrt{(2 \gamma+1)^{2}+4 \gamma \xi}\right] 2\right\} d \xi, \\
& X>Y>0 \text {, } \\
& f_{0}(\xi)=\frac{\xi}{\xi+2 \gamma+1-\sqrt{(2 \gamma+1)^{2}+4 \gamma \xi}}, \\
& p(n, n) \sim \frac{\varepsilon^{2}}{2 \pi i} \int_{\mathrm{Br}_{+}} h_{0}(\xi) e^{Y \xi} d \xi \\
& h_{0}(\xi)=\frac{(2-\nu) \xi+(2 \gamma+1)(1-\nu)-(1-\nu) \sqrt{(2 \gamma+1)^{2}+4 \gamma \xi}}{(2-\nu)\left[\xi+2 \gamma+1-\sqrt{(2 \gamma+1)^{2}+4 \gamma \xi}\right]}, \\
& p(m, n) \sim \varepsilon\left(\frac{\varepsilon \gamma}{2}\right)^{n-m} \frac{1}{2 \pi i} \int_{\mathrm{Br}_{+}} \bar{g}(\xi) e^{Y \xi} d \xi, \quad n>m, \\
& \bar{g}(\varepsilon)=\frac{\nu}{2-\nu} \frac{1}{\gamma} \frac{\sqrt{(2 \gamma+1)^{2}+4 \gamma \xi}-(2 \gamma+1)}{2 \gamma+1+\xi-\sqrt{(2 \gamma+1)^{2}+4 \gamma \xi}} d \xi
\end{aligned}
$$

Here $\mathrm{Br}_{+}$is a vertical contour in the $\xi$-plane, which lies to the right of all singularities.

The expression in (78) applies for $m>n, m<n$, and $m=n$, and in the light traffic limit the discontinuity of $p(m, n)$ along the diagonal will appear only in the higher order terms. In HTL1, (79)-(81) show a piecewise geometric distribution in the $\ell$ variable, and an exponential density in $Y$. For HTL2, writing (82) as $p(m, n) \sim \varepsilon^{2} \mathscr{F}(X, Y)$ we can easily show that $\int_{0}^{\infty} \int_{Y}^{\infty} \mathscr{F}(X, Y) d X d Y=1$ so that to leading order the probability mass concentrates where $m, n=O\left(\varepsilon^{-1}\right)$ with $X>Y$. From (84) we have $p(n, n)=O\left(\varepsilon^{2}\right)$ but the total mass along the main diagonal is $O(\varepsilon)$, which is smaller than the mass in (82). Then also $p(n-1, n)=O\left(\varepsilon^{2}\right)$ with total mass $\sum_{n} p(n-1, n)=O(\varepsilon)$, which is comparable to that along the main diagonal. The diagonals with $n-m \geqslant 2$ have mass $O\left(\varepsilon^{n-m}\right)$, which is smaller still. The integrands in (82), (84), and (86) have branch points at $\xi=-(2 \gamma+1)^{2} /(4 \gamma)$, are analytic at $\xi=0$, and may have poles at $\xi=-2$, if $\gamma>1 / 2$.

This completes our summarization of the exact and asymptotic results. Despite the seeming complexity and the many separate cases, all the results follow from fairly standard asymptotic evaluations of the integrals in Theorems 1, as we will show in Sections 4 and 5. 


\section{The Exact Solution}

We solve the difference equation(s) in (1)-(9) and thus obtain Theorem 1 . We begin by introducing the three generating functions:

$$
\begin{gathered}
F(z, w)=\sum_{n=0}^{\infty} \sum_{m=n+1}^{\infty} z^{m-n-1} w^{n} p(m, n) \\
G(z, w)=\sum_{n=1}^{\infty} \sum_{m=0}^{n-1} z^{n-m-1} w^{n} p(m, n) \\
H(w)=\sum_{n=0}^{\infty} p(n, n) w^{n}
\end{gathered}
$$

and we note that $H(0)=p(0,0)$, and

$$
\begin{aligned}
& F(0, w)=\sum_{n=0}^{\infty} p(n+1, n) w^{n} \\
& G(0, w)=\sum_{n=1}^{\infty} p(n-1, n) w^{n}
\end{aligned}
$$

Then (9) shows that

$$
F(1,1)+G(1,1)+H(1)=1 .
$$

From (1) and (3) we obtain, after some calculation,

$$
\begin{aligned}
{\left[\rho_{1}+\right.} & \left.\rho_{2}+1-\rho_{1} z-\frac{1}{z}-\rho_{2} \frac{w}{z}\right] F(z, w) \\
= & \rho_{1} H(w)+(1-v) \frac{H(w)-p(0,0)}{w} \\
& -\frac{1+\rho_{2} w}{z} F(0, w)
\end{aligned}
$$

and (2) and (4) lead to

$$
\begin{aligned}
{\left[\rho_{1}+\right.} & \left.\rho_{2}+1-\frac{\rho_{1}}{z}-\rho_{2} w z-\frac{1}{w z}\right] G(z, w) \\
= & \rho_{2} w H(w)+v[H(w)-p(0,0)] \\
& -\frac{1}{z}\left(\rho_{1}+\frac{1}{w}\right) G(0, w) .
\end{aligned}
$$

Equation (5) along $m=n$ then leads to the following relation between $F(0, w), G(0, w)$, and $H(w)$ :

$$
\begin{aligned}
\left(\rho_{1}-\right. & \left.\rho_{2}+1\right) H(w)-p(0,0) \\
& =\left(\rho_{1}+\frac{1}{w}\right) G(0, w)+\left(\rho_{2} w+1\right) F(0, w) .
\end{aligned}
$$

If we define

$$
\mathcal{S}(\xi)=\sum_{N=0}^{\infty}\left[\sum_{m+n=N} p(m, n)\right] \xi^{N},
$$

then from (96) and (88)-(90) we find that

$$
\mathcal{S}(\xi)=H\left(\xi^{2}\right)+\xi F\left(\xi, \xi^{2}\right)+\xi^{-1} G\left(\xi^{-1}, \xi^{2}\right) .
$$

Using (93) with $(z, w)=\left(\xi, \xi^{2}\right),(94)$ with $(z, w)=\left(\xi^{-1}, \xi^{2}\right)$ and (95) with $w=\xi^{2}$, we obtain for $\delta(\xi)$ the simpler equation

$$
\left[\rho_{1}+\rho_{2}+1-\left(\rho_{1}+\rho_{2}\right) \xi-\frac{1}{\xi}\right] \mathcal{S}(\xi)=\left(1-\frac{1}{\xi}\right) p(0,0)
$$

so that $\mathcal{S}(\xi)=p(0,0) /\left[1-\left(\rho_{1}+\rho_{2}\right) \xi\right]$ and then $\mathcal{S}(1)=1$ by (92), and thus $p(0,0)=1-\rho_{1}-\rho_{2}$. Then from (96) we have

$$
\sum_{m+n=N} p(m, n)=\left(1-\rho_{1}-\rho_{2}\right)\left(\rho_{1}+\rho_{2}\right)^{N}
$$

so that $N_{1}+N_{2}$ follows a geometric distribution with parameter $\rho_{1}+\rho_{2}$, and hence $N_{1}+N_{2}$ behaves as the standard $M / M / 1$ model.

The coefficient of $F(z, w)$ in (93) has roots at

$$
\begin{aligned}
z & =\frac{1}{2 \rho_{1}}\left[1+\rho_{1}+\rho_{2} \pm \sqrt{\left(\rho_{1}+\rho_{2}+1\right)^{2}-4 \rho_{1}\left(\rho_{2} w+1\right)}\right] \\
& \equiv Z_{ \pm}(w)
\end{aligned}
$$

while that of $G(z, w)$ in (94) has roots at

$$
\begin{aligned}
z & =\frac{1}{2 \rho_{2} w}\left[1+\rho_{1}+\rho_{2} \pm \sqrt{\left(\rho_{1}+\rho_{2}+1\right)^{2}-4 \rho_{2}\left(\rho_{1} w+1\right)}\right] \\
& \equiv \frac{Z_{ \pm}^{*}(w)}{w} .
\end{aligned}
$$

By using (95) to express $H(w)$ in terms of $F(0, w)$ and $G(0, w)$, eliminating $H(w)$ in the right sides of (93) and (94), and requiring that $F(z, w)$ be analytic at $z=Z_{-}(w)$ and that $G(z, w)$ be analytic at $z=Z_{-}^{*}(w) / w$, we obtain two equations for $F(0, w)$ and $G(0, w)$ :

$$
\begin{aligned}
& \frac{\left(\rho_{2} w+1\right)\left(\rho_{2} w+v\right)}{\rho_{1}+\rho_{2}+1} F(0, w) \\
& +\left[\frac{\left(\rho_{1} w+1\right)\left(\rho_{2} w+v\right)}{w\left(\rho_{1}+\rho_{2}+1\right)}-\frac{\rho_{1} w+1}{Z_{-}^{*}(w)}\right] G(0, w) \\
& =p(0,0)\left[\nu-\frac{\rho_{2} w+v}{\rho_{1}+\rho_{2}+1}\right], \\
& {\left[\frac{\left(\rho_{2} w+1\right)\left(\rho_{1} w+1-v\right)}{\left(\rho_{1}+\rho_{2}+1\right) w}-\frac{1+\rho_{2} w}{Z-(w)}\right] F(0, w)} \\
& \quad+\frac{\left(\rho_{1} w+1\right)\left(\rho_{1} w+1-\nu\right)}{w^{2}\left(\rho_{1}+\rho_{2}+1\right)} G(0, w) \\
& =p(0,0)\left[\frac{1-\nu}{w}-\frac{\rho_{1} w+1-\nu}{\left(\rho_{1}+\rho_{2}+1\right) w}\right] .
\end{aligned}
$$


Solving the algebraic system in (98) and (99), and then using the result in (95) to compute $H(w)$, we hence obtain explicitly the right sides of (93) and (94). In particular,

$$
\begin{aligned}
F(z, w) & =\left(1-\rho_{1}-\rho_{2}\right) \frac{f(w)}{Z_{+}(w)-z} \\
& =\left(1-\rho_{1}-\rho_{2}\right)\left\{\sum_{J=0}^{\infty} \frac{z^{J}}{\left[Z_{+}(w)\right]^{J+1}}\right\} f(w),
\end{aligned}
$$

where $f(w)$ is as in (12)-(14). Comparing (100) to (88) we conclude that

$$
\sum_{n=0}^{\infty} p(n+1+J, n) w^{n}=\left(1-\rho_{1}-\rho_{2}\right) \frac{f(w)}{\left[Z_{+}(w)\right]^{J+1}}
$$

for $J \geqslant 0$. Setting $J=m-n-1$ and inverting the generating function in (101) leads to (10) for $m>n$. Similarly, inverting the double generating function for $G(z, w)$ in (89) leads to (15), and we note that (10) and (15) are consistent with the symmetry $\left(n, \rho_{1}, v, Z_{+}, f\right) \leftrightarrow\left(m, \rho_{2}, 1-v, Z_{+}^{*}, g\right)$. Finally, $p(n, n)$ in (19) is obtained by inverting the generating function $H(w)=\left(1-\rho_{1}-\rho_{2}\right) h(w)$. We have thus established Theorem 1 .

\section{Asymptotics of the Joint Distribution}

We derive Theorems 2-4 by expanding asymptotically the integrals in Theorem 1 . We will use a combination of the saddle point method and singularity analysis. Good general references on techniques for asymptotically evaluating integrals can be found in [17-21].

We need to understand the singularities of the integrands in (10), (15), and (19). There are clearly branch points where $w=w_{b}$ and $w=w_{b}^{*}$, with

$$
\begin{aligned}
& w_{b}=\frac{\left(\rho_{1}+\rho_{2}+1\right)^{2}-4 \rho_{1}}{4 \rho_{1} \rho_{2}}, \\
& w_{b}^{*}=\frac{\left(\rho_{1}+\rho_{2}+1\right)^{2}-4 \rho_{2}}{4 \rho_{1} \rho_{2}} .
\end{aligned}
$$

Since $\rho_{1} \geqslant \rho_{2}$ we have $w_{b} \leqslant w_{b}^{*}$ and for $\rho_{1}>\rho_{2}$ the branch point at $w_{b}^{*}$ is farther from the origin than the one at $w_{b}$. In fact, $w_{b}^{*}$ will never play a role in the asymptotics. The integrands are also singular at $w=0$, where (10) has a pole of order $n+1$, and (15) has a pole of order $m+1$. The only possible other singular points are at the zeros of den $(w)$. We can easily verify that $w=1$ is a simple zero of all four functions $\operatorname{num}_{f}(w), \operatorname{num}_{g}(w), \operatorname{num}(w)$, and $\operatorname{den}(w)$, so all the integrands are analytic at $w=1$. In the appendix we study in detail the algebraic equation $\operatorname{den}(w)=0$, and show that the only possible zero is at $w=w_{p}$

$$
w_{p}=\left(\rho_{1}+\rho_{2}\right)^{-2}, \quad\left(\rho_{1}+\rho_{2}\right)^{2}>\rho_{1}-\rho_{2},
$$

but only if $\left(\rho_{1}+\rho_{2}\right)^{2}>\rho_{1}-\rho_{2}$ (if $\left(\rho_{1}+\rho_{2}\right)^{2}=\rho_{1}-\rho_{2}$ then $\operatorname{den}(w)$ has a branch point at $\left.w=w_{p}\left(=w_{b}\right)\right)$. If $\left(\rho_{1}+\rho_{2}\right)^{2}<$ $\rho_{1}-\rho_{2}$ then the two branch points are the only singularities of the functions $f(w), g(w)$, and $h(w)$ in (12), (16), and (19). If $\left(\rho_{1}+\rho_{2}\right)^{2}>\rho_{1}-\rho_{2}$, which is clearly true in the symmetric case, then $w=w_{p}$ is a simple pole of these functions (since $\left.\operatorname{den}^{\prime}\left(w_{p}\right) \neq 0\right)$. In view of (105) and (106), we have $1<w_{p}<$ $w_{b}<w_{b}^{*}$, if the pole is present and the stability condition $\rho_{1}+\rho_{2}<1$ holds.

Consider first (19). If $\left(\rho_{1}+\rho_{2}\right)^{2}>\rho_{1}-\rho_{2}$ the pole at $w_{p}$ determines the asymptotic behavior, as it is closer to $w=0$ than the branch point at $w_{b}$. Hence as $n \rightarrow \infty$

$$
\begin{aligned}
p(n, n) & \sim-\left(1-\rho_{1}-\rho_{2}\right) \lim _{w \rightarrow w_{p}}\left[\frac{\left(w-w_{p}\right) h(w)}{w^{n+1}}\right] \\
& =-\left(1-\rho_{1}-\rho_{2}\right) w_{p}^{-n-1} \frac{\operatorname{num}\left(w_{p}\right)}{\operatorname{den}^{\prime}\left(w_{p}\right)} .
\end{aligned}
$$

Straightforward computations, using (14) and (21), show that

$$
\begin{aligned}
& \operatorname{den}^{\prime}\left(w_{p}\right) \\
& =\frac{2 \rho_{1} \rho_{2}\left(\rho_{1}+\rho_{2}\right)^{2}\left[\left(\rho_{1}+\rho_{2}\right)^{2}+\rho_{2} \nu+\rho_{1}(1-\nu)\right]\left(1-\rho_{1}-\rho_{2}\right)}{\left(\rho_{1}+\rho_{2}\right)^{4}-\left(\rho_{1}-\rho_{2}\right)^{2}} \\
& \operatorname{num}\left(w_{p}\right)=\frac{-2 \rho_{1} \rho_{2}\left(1-\rho_{1}-\rho_{2}\right)}{\left(\rho_{1}+\rho_{2}\right)^{2}} . \\
& w^{-n}\left[Z_{+}(w)\right]^{n-m}=\exp \left\{n\left[(1-R) \log \left(Z_{+}(w)\right)-\log w\right]\right\}
\end{aligned}
$$

and let $F(w)=F(w ; R)=(1-R) \log \left(Z_{+}(w)\right)-\log w$. Then with (110), (10) has saddle points where $F^{\prime}(w)=0$, or

$$
(1-R) \frac{Z_{+}^{\prime}(w)}{Z_{+}(w)}-\frac{1}{w}=0 .
$$

Solving (111) using (11), after some algebra we are led to the saddle $w_{s}=w_{s}(R)$ in (28). There is also a second saddle at $\bar{w}_{s}$, where $\bar{w}_{s}$ corresponds to replacing $\sqrt{\cdots}$ in $(28)$ by $-\sqrt{\cdots}$. By evaluating $F^{\prime \prime}\left(w_{s}\right)$ and $F^{\prime \prime}\left(\bar{w}_{s}\right)$ we can easily show that the directions of steepest descent at the saddle $(\mathrm{s}) \operatorname{are} \arg \left(w-w_{s}\right)=$ $\pm \pi / 2$ and $\arg \left(w-\bar{w}_{s}\right)=0, \pi$. From (28) we have $w_{s}(1)=w_{b}$, so as $R=m / n \downarrow 1$ the saddle approaches a branch point. Also, $w_{s} \rightarrow 0$ as $R \rightarrow \infty$. It is possible for the pole and saddle to coalesce. Setting $w_{s}(R)=w_{p}=\left(\rho_{1}+\rho_{2}\right)^{-2}$ and solving this equation for $R$ we find, again after some algebra, that

$$
w_{s}(R)=w_{p} \quad \text { when } R=R_{c}
$$

where $R_{c}$ is given by (26). If $R>R_{c}(>1)$, we have $w_{s}<$ $w_{p}$ and if $R \in\left(1, R_{c}\right)$, we have $w_{s}>w_{p}$. From (28) we can 
show that $w_{s}^{\prime}(R)<0$ so that the saddle location decreases with the queue length ratio $m / n=R$. If $R>R_{c}$ we deform the contour in (10), which is a small counterclockwise loop about $z=0$, into a saddle point contour, on which $|w|=w_{s}(R)$. Then the new contour traverses the saddle in the direction(s) of steepest descent and the standard Laplace estimate of (10) is

$$
p(m, n) \sim \frac{1-\rho_{1}-\rho_{2}}{2 \pi} \sqrt{\frac{2 \pi}{n F^{\prime \prime}\left(w_{s}\right)}} \frac{f\left(w_{s}\right)}{w_{s}} \exp \left[n F\left(w_{s}\right)\right] .
$$

But $e^{n F\left(w_{s}\right)}=w_{s}^{-n}\left[Z_{+}\left(w_{s}\right)\right]^{n-m}$ and, in view of (111),

$$
F^{\prime \prime}(w)=\frac{1}{w^{2}}+(1-R)\left[\frac{Z_{+}^{\prime \prime}(w)}{Z_{+}(w)}-\left(\frac{Z_{+}^{\prime}(w)}{Z_{+}(w)}\right)^{2}\right] .
$$

Then using (11) we commute $w_{s}^{2} F^{\prime \prime}\left(w_{s}\right)$ to find that $w_{s}^{2}(R) F^{\prime \prime}\left(w_{s}(R)\right)$ is the same as $M(R)$ in (30). Thus (113) is the same as the right side of (27), and we have established the asymptotics of $p(m, n)$ for $R>R_{c}$.

If $1<R<R_{c}, w_{p}>w_{s}$ and in deforming the loop in (10) to the saddle point contour $|w|=w_{s}(R)$ we must take into account the contribution from the residue at the pole $w_{p}$. But we have $F\left(w_{p}\right) \geqslant F\left(w_{s}\right)$ with equality only if $R=R_{c}$, when $w_{p}=w_{s}$. Thus the pole contribution dominates the saddle contribution and we have

$$
p(m, n) \sim-\left(1-\rho_{1}-\rho_{2}\right) w_{p}^{-n-1}\left[Z_{+}\left(w_{p}\right)\right]^{n-m} \frac{\operatorname{num}_{f}\left(w_{p}\right)}{\operatorname{den}^{\prime}\left(w_{p}\right)} .
$$

From (13) we find that

$$
\begin{aligned}
\operatorname{num}_{f}\left(w_{p}\right)= & \frac{-2 \rho_{2}}{\left(\rho_{1}+\rho_{2}\right)^{2}}\left[\left(\rho_{1}+\rho_{2}\right)^{2}(1-\nu)+\rho_{1}\right] \\
& \times\left(1-\rho_{1}-\rho_{2}\right)
\end{aligned}
$$

and from (11)

$$
\begin{aligned}
& Z_{+}\left(w_{p}\right) \\
& =\frac{1}{2 \rho_{1}}\left[\rho_{1}+\rho_{2}+1+\sqrt{\left(\rho_{1}+\rho_{2}+1\right)^{2}-4 \rho_{1}-\frac{4 \rho_{1} \rho_{2}}{\left(\rho_{1}+\rho_{2}\right)^{2}}}\right] \\
& =\frac{\left(\rho_{1}+\rho_{2}\right)^{2}+\rho_{2}}{\rho_{1}\left(\rho_{1}+\rho_{2}\right)},
\end{aligned}
$$

when $\left(\rho_{1}+\rho_{2}\right)^{2}+\rho_{2}-\rho_{1}>0$. With (115)-(117) and (108) we have established (34) for $R \in\left(1, R_{c}\right)$, where $e^{n F\left(w_{s} ; R\right)}$ gives the product form expression and $\left(1-\rho_{1}-\right.$ $\left.\rho_{2}\right)\left|\operatorname{num}_{f}\left(w_{p}\right)\right| /\left[w_{p} \operatorname{den}^{\prime}\left(w_{p}\right)\right]$ corresponds to the multiplicative constant $C_{+}$.

As $R \rightarrow \infty$ we have $w_{s} \rightarrow 0$ and we must then reconsider the asymptotics of (10). From (28) $w_{s}=O\left(R^{-1}\right)$ as $R \rightarrow \infty$ so we scale $w=u / m$ in the integral in (10) and consider the limit $m \rightarrow \infty$ with $n=O(1)$. Then

$$
\begin{aligned}
{\left[Z_{+}(w)\right]^{n-m} } & =\left[Z_{+}(0)+Z_{+}^{\prime}(0) \frac{u}{m}+O\left(m^{-2}\right)\right]^{n-m} \\
& \sim\left[Z_{+}(0)\right]^{n-m} \exp \left(-\frac{Z_{+}^{\prime}(0)}{Z_{+}(0)} u\right)
\end{aligned}
$$

and (10) becomes asymptotically

$$
\begin{aligned}
p(m, n) \sim & \frac{\left(1-\rho_{1}-\rho_{2}\right)}{2 \pi i} f(0)\left[Z_{+}(0)\right]^{n-m} \\
& \times \oint \frac{m^{n}}{u^{n+1}} \exp \left[-\frac{Z_{+}^{\prime}(0)}{Z_{+}(0)} u\right] d u \\
= & \left(1-\rho_{1}-\rho_{2}\right) f(0)\left[Z_{+}(0)\right]^{n-m}\left(-\frac{Z_{+}^{\prime}(0)}{Z_{+}(0)}\right)^{n} \frac{m^{n}}{n !} .
\end{aligned}
$$

Since $Z_{+}^{\prime}(0)=-\rho_{2} / \sqrt{\left(\rho_{1}+\rho_{2}+1\right)^{2}-4 \rho_{1}}$ we see that (119) is the same as (25).

Now consider $R=m / n \approx R_{c}$, where the saddle and pole are close to each other. This is a standard problem that is discussed, for example, in $[17,18]$. We now expand the integrand in (10) about $w=w_{p}$, ultimately scaling $w-w_{p}=$ $O\left(n^{-1 / 2}\right)$, and then the integrand will approach a limiting value as $n \rightarrow \infty$. We have, by Taylor/Laurent series,

$$
\begin{aligned}
f(w)= & \frac{\operatorname{num}_{f}\left(w_{p}\right)}{\operatorname{den}^{\prime}\left(w_{p}\right)} \frac{1}{w-w_{p}}+O(1), \quad w \longrightarrow w_{p}, \\
n F(w)= & -n \log w_{p}-(m-n) \log \left[Z_{+}\left(w_{p}\right)\right] \\
& -\left[\frac{n}{w_{p}}+\frac{m-n}{Z_{+}\left(w_{p}\right)} Z_{+}^{\prime}\left(w_{p}\right)\right]\left(w-w_{p}\right) \\
& +\frac{1}{2}\left\{\frac{n}{w_{p}^{2}}+(m-n)\left[\frac{Z_{+}^{\prime}\left(w_{p}\right)}{\left.Z_{+}\left(w_{p}\right)\right]}\right]^{2}\right. \\
& \left.-(m-n) \frac{Z_{+}^{\prime \prime}\left(w_{p}\right)}{Z_{+}\left(w_{p}\right)}\right\}\left(w-w_{p}\right)^{2} \\
& O\left(n\left(w-w_{p}\right)^{3}\right) .
\end{aligned}
$$

From (11) and (122) we find that

$$
\frac{Z_{+}^{\prime}\left(w_{p}\right)}{Z_{+}\left(w_{p}\right)}=\left.\frac{1}{w_{p}} \frac{n}{n-m}\right|_{R=R_{c}}=\frac{1}{w_{p}} \frac{1}{1-R_{c}},
$$

since, at $R=R_{c}, w_{p}=w_{s}$. It follows that

$$
-\frac{n}{w_{p}}-\frac{m-n}{Z_{+}\left(w_{p}\right)} Z_{+}^{\prime}\left(w_{p}\right)=\frac{n}{w_{p}} \frac{R-R_{c}}{R_{c}-1}=\frac{\sqrt{n} \xi}{w_{p}\left(R_{c}-1\right)},
$$


where $\xi$ was defined above (31). Also, from (11) we find that

$$
Z_{+}^{\prime \prime}\left(w_{p}\right)=-\frac{2 \rho_{1} \rho_{2}^{2}\left(\rho_{1}+\rho_{2}\right)^{3}}{\left[\left(\rho_{1}+\rho_{2}\right)^{2}+\rho_{2}-\rho_{1}\right]^{3}} .
$$

Then setting

$$
w-w_{p}=\frac{1}{\sqrt{n}} w_{p}\left[\frac{R_{c}}{R_{c}-1}+\frac{2 \rho_{1} \rho_{2}}{\left(\left(\rho_{1}+\rho_{2}\right)^{2}+\rho_{2}-\rho_{1}\right)^{2}}\right]^{-1 / 2} v
$$

and scaling $\xi$ as in (32), (121) becomes

$$
\begin{aligned}
n F(w)= & -n \log w_{p}-(m-n) \log \left[Z_{+}\left(w_{p}\right)\right] \\
& +\xi_{1} v+\frac{1}{2} v^{2}+O\left(n^{-1 / 2}\right) .
\end{aligned}
$$

Then we use (120) and (126) to get

$$
\begin{aligned}
p(m, n)= & w_{p}^{-n}\left[Z_{+}\left(w_{p}\right)\right]^{n-m} \\
& \times\left(-C_{+}\right) \frac{1}{2 \pi i} \int_{\mathrm{Br}_{-}} e^{\xi_{1} v} e^{v^{2} / 2} \frac{1}{v} d v \\
& \times\left[1+O\left(n^{-1 / 2}\right)\right],
\end{aligned}
$$

where $\mathrm{Br}_{-}$is a vertical contour with $\operatorname{Re}(v)<0$. To obtain (127) we shifted the original contour in (10) into the circle $|w|=$ $w_{p}-\varepsilon_{1}, \varepsilon_{1}>0$, and note that $|w|<w_{p}$ implies to leading order that $\operatorname{Re}(v)<0$. The integral in (127) can be expressed in terms of a parabolic cylinder function of order $p=-1$ (see (61)), which can be expressed in terms of the standard error function, using the identity

$$
-\frac{1}{2 \pi i} \int_{\mathrm{Br}_{-}} \frac{1}{v} e^{\xi_{1} v} e^{v^{2} / 2} d v=\frac{1}{\sqrt{2 \pi}} \int_{\xi_{1}}^{\infty} e^{-u^{2} / 2} d u .
$$

With (128) and (117), (127) becomes the same as (31), so we have derived the leading term for the range $R-R_{c}=O\left(n^{-1 / 2}\right)$. We have thus covered all of the necessary ranges that have $R \geqslant 1$, and established items (i) $-(\mathrm{v})$ in Theorem 2 .

Now we consider ranges with $R<1(m<n)$ where $p(m, n)$ is given by the integral in (15). The analysis is completely analogous to the expansion of (10), so we merely sketch the details. The function $g(w)$ in (16) has a simple pole at $w=w_{p}=\left(\rho_{1}+\rho_{2}\right)^{-2}>1$ if $\left(\rho_{1}+\rho_{2}\right)^{2}>\rho_{1}-\rho_{2}$, with residue $\operatorname{num}_{g}\left(w_{p}\right) / \operatorname{den}^{\prime}\left(w_{p}\right)$ where

$$
\operatorname{num}_{g}\left(w_{p}\right)=-\frac{2 \rho_{1}}{\left(\rho_{1}+\rho_{2}\right)^{2}}\left[\left(\rho_{1}+\rho_{2}\right)^{2} v+\rho_{2}\right]\left(1-\rho_{1}-\rho_{2}\right) \text {. }
$$

We write $w^{-m}\left[Z_{+}^{*}(w)\right]^{m-n}=\exp \left[n F_{*}(w ; R)\right]$ where

$$
F_{*}(w ; R)=-R \log w+(R-1) \log \left[Z_{+}^{*}(w)\right] .
$$

Thus for $m, n$ simultaneously large $F_{*}$ will have a saddle where $F_{*}^{\prime}(w)=0$, and this leads to $w=w_{s}^{*}=w_{s}^{*}(R)$, as in (43). The saddle and pole coalesce where $R=R_{c}^{*}(<1)$ in (37). For $R \in\left(R_{c}^{*}, 1\right)$ we have $w_{p}<w_{s}^{*}(R)$ and the pole determines the asymptotic behavior of $p(m, n)$. Then

$$
p(m, n) \sim-\left(1-\rho_{1}-\rho_{2}\right) \frac{\operatorname{num}_{g}\left(w_{p}\right)}{\operatorname{den}^{\prime}\left(w_{p}\right)} w_{p}^{-m-1}\left[Z_{+}^{*}\left(w_{p}\right)\right]^{m-n},
$$

and this leads to (38) with (39), as now $Z_{+}^{*}\left(w_{p}\right)=\left[\left(\rho_{1}+\rho_{2}\right)^{2}+\right.$ $\left.\rho_{1}\right] /\left[\rho_{2}\left(\rho_{1}+\rho_{2}\right)\right]$. For $R \in\left(0, R_{c}^{*}\right)$ the saddle determines the asymptotics, and the estimate

$$
p(m, n) \sim \frac{1-\rho_{1}-\rho_{2}}{\sqrt{2 \pi n}} \sqrt{\frac{1}{F_{*}^{\prime \prime}\left(w_{s}^{*}\right)}} \frac{g\left(w_{s}^{*}\right)}{w_{s}^{*}} \exp \left[n F_{*}\left(w_{s}^{*}\right)\right]
$$

leads to (42), with (43)-(45). As $R \rightarrow 0$ the saddle $w_{s}^{*} \rightarrow 0$ and the result in (46) can be obtained by approximating the integrand in (15) for $w=O\left(n^{-1}\right)$, similarly as in (118) and (119) (with $Z_{+}$replaced by $Z_{+}^{*}, f$ by $g$, and $m$ by $n$ ). Thus we obtain (46). Finally, when $R \approx R_{c}^{*}$ the pole and saddle are close. Then we scale $R-R_{c}^{*}=\eta / \sqrt{n}=O\left(n^{-1 / 2}\right)$ and expand the integrand in (15) near $w=w_{p}$, with $w-w_{p}=O\left(n^{-1 / 2}\right)$. This leads ultimately to (40) with (41). We have thus sketched the derivation of items (vi)-(ix) in Theorem 2.

Next we take $\left(\rho_{1}+\rho_{2}\right)<\rho_{1}-\rho_{2}(>0)$ and establish Theorem 3. Now the pole at $w=w_{p}$ is absent. We first consider the diagonal probabilities in (19). The only singularities of $h(w)$ as the branch points at $w_{b}$ and $w_{b}^{*}$, and the former determines the large $n$ asymptotics, as $w_{b}<w_{b}^{*}$ in view of (105). Expanding $h(w)$ about $w=w_{b}$ will transfer immediately to an asymptotic series for $p(n, n)$, but here we focus on the leading term. From (14) and (21) we have

$$
\begin{gathered}
\operatorname{num}(w)=A+B \sqrt{w_{b}-w}+O\left(w-w_{b}\right), \\
\operatorname{den}(w)=C+D \sqrt{w_{b}-w}+O\left(w-w_{b}\right),
\end{gathered}
$$

where

$$
\begin{gathered}
A=-2 \rho_{1} \rho_{2} w_{b}+\left(\rho_{1}+\rho_{2}+1\right)\left[\rho_{1} \nu+\rho_{2}(1-v)\right] \\
-2 v \rho_{1} \sqrt{\rho_{1}-\rho_{2}} \\
B=-2 \sqrt{\rho_{1} \rho_{2}} \rho_{2}(1-\nu) \\
C=\left(\rho_{1}+\rho_{2}+1\right)\left[\rho_{1} \nu+\rho_{2}(1-v)\right] \\
-2 \rho_{1}\left(\rho_{2} w_{b}+\nu\right) \sqrt{\rho_{1}-\rho_{2}} \\
D=-2 \sqrt{\rho_{1} \rho_{2}}\left[\rho_{1} \rho_{2} w_{b}+\rho_{2}(1-v)\right]
\end{gathered}
$$

Note that $\sqrt{\left(\rho_{1}+\rho_{2}+1\right)^{2}-4 \rho_{2}\left(\rho_{1} w_{b}+1\right)}=2 \sqrt{\rho_{1}-\rho_{2}}$. Using (133)-(137) we have

$$
h(w)=\frac{A}{C}+\frac{B C-A D}{C^{2}} \sqrt{w_{b}-w}+O\left(w-w_{b}\right) .
$$


The expansion of $h(w)$ will be in powers of $\sqrt{w_{b}-w}$, but only the odd powers will contribute to the asymptotics. Now,

$$
\begin{aligned}
\frac{1}{2 \pi i} \oint \frac{\sqrt{w_{b}-w}}{w^{n+1}} d w & =\sqrt{w_{b}} w_{b}^{-n} \frac{1}{2 \pi i} \oint \frac{\sqrt{1-U}}{U^{n+1}} d U \\
& =-\sqrt{w_{b}} w_{b}^{-n} \frac{1}{2 n-1} \frac{(2 n) !}{4^{n}(n !)^{2}} \\
& \sim-\frac{1}{2} \sqrt{\frac{w_{b}}{\pi}} n^{-3 / 2} w_{b}^{-n}, \quad n \longrightarrow \infty,
\end{aligned}
$$

where we used the binomial expansion of $\sqrt{1-U}$ and Stirling's formula. It follows that the leading term for $p(n, n)$ is, in view of (138) and (139),

$$
p(n, n) \sim-\frac{1}{2} \sqrt{\frac{w_{b}}{\pi}} \frac{B C-A D}{C^{2}} n^{-3 / 2} w_{b}^{-n},
$$

with a correction that is $O\left(n^{-1}\right)$ relative to the leading term, which may be computed from the $O\left(\left(w_{b}-w\right)^{3 / 2}\right)$ term in (138), and a refined Stirling approximation of the factorials in (139). Some of the algebra in our calculations is simplified by introducing $a=\rho_{1}+\rho_{2}$ and $b=\sqrt{\rho_{1}-\rho_{2}}$. Then $C$ in (136) factors as

$$
\begin{aligned}
C= & (a-b)(b-1)\left[b\left(v-\frac{1}{2}\right)-\frac{1}{2}(a+b+1)\right] \\
= & -\left[\sqrt{\rho_{1}-\rho_{2}}-\left(\rho_{1}+\rho_{2}\right)\right] \\
& \times\left[1-\sqrt{\rho_{1}-\rho_{2}}\right]\left[\frac{1}{2}\left(\rho_{1}+\rho_{2}+1\right)+\sqrt{\rho_{1}-\rho_{2}}(1-v)\right],
\end{aligned}
$$

and $C<0$ if $\left(\rho_{1}+\rho_{2}\right)^{2}<\rho_{1}-\rho_{2}$. After some calculation we find that

$$
\begin{aligned}
B C-A D= & 2\left(\rho_{1} \rho_{2}\right)^{3 / 2}\left(1-\sqrt{\rho_{1}-\rho_{2}}\right)^{2} w_{b} \\
& \times\left[\left(\rho_{1}+\rho_{2}\right)\left(\nu-\frac{1}{2}\right)-\frac{1}{2}-\sqrt{\rho_{1}-\rho_{2}}\right]
\end{aligned}
$$

so that $B C-A D<0$ and then $(A D-B C) /\left(2 C^{2}\right)$ is the same as the constant $\bar{C}$ in (53). We have thus established (52).

Next we consider $R=m / n>1$. The saddle point calculation that led to (27) does not depend on whether $\left(\rho_{1}+\right.$ $\left.\rho_{2}\right)^{2} \gtrless \rho_{1}-\rho_{2}$. If the pole at $w_{p}$ is absent, then (27) will apply for all $R \in(1, \infty)$. For $n=O(1)$ and $m \rightarrow \infty$ (25) again holds, since this calculation is independent of whether $w_{p}$ exists or not. However, now a different analysis is needed for $R \approx 1$, and the appropriate scale is to fix $m-n$ and let $n \rightarrow \infty$. As $R \downarrow 1$ we have $w_{s}(R) \rightarrow w_{s}(1)=w_{b}$ and then

$$
Z_{+}\left(w_{s}\right) \longrightarrow Z_{+}\left(w_{b}\right)=\frac{\rho_{1}+\rho_{2}+1}{2 \rho_{1}} .
$$

The expansion in (27) breaks down as $R \rightarrow 1$ (if $\left(\rho_{1}+\rho_{2}\right)^{2}<$ $\left.\rho_{1}-\rho_{2}\right)$ as (30) shows that

$$
M(R) \sim \frac{2}{(R-1)^{2}} \frac{\left(\rho_{1}+\rho_{2}+1\right)^{2}}{\left(\rho_{1}+\rho_{2}+1\right)^{2}-4 \rho_{1}}, \quad R \downarrow 1
$$

and thus the factor $1 / \sqrt{M(R)}$ in (27) vanishes linearly as $R \downarrow$ 1 , which indicates a problem in the asymptotics.

We thus reexamine (10) for $m-n=O(1)$ and $n$ large. We again employ singularity analysis and expand the integrand about the branch point $w_{b}$. We let

$$
\begin{aligned}
\operatorname{num}_{f}(w) & =A_{f}+B_{f} \sqrt{w_{b}-w}+O\left(w-w_{b}\right) \\
& =A_{f}+O\left(w-w_{b}\right),
\end{aligned}
$$

since $B_{f}=0$ in view of (13). Then

$$
f(w)=\frac{A_{f}}{C}-\frac{A_{f} D}{C^{2}} \sqrt{w_{b}-w}+O\left(w-w_{b}\right)
$$

where

$$
\begin{aligned}
A_{f}= & -2 \rho_{1} \rho_{2} w_{b}+\rho_{1}^{2}(1-v)+\rho_{1} \rho_{2} \\
& -\rho_{2}(1-v)+\left(\rho_{1}^{2}+\rho_{1}\right) \nu \\
& +2\left[\rho_{2}-\left(\rho_{1}+\rho_{2}\right) \nu\right] \sqrt{\rho_{1}-\rho_{2}} \\
= & (1-b)^{2}\left[v a-\frac{1}{2}(a+1)-b\right] \\
= & \left(1-\sqrt{\rho_{1}-\rho_{2}}\right)^{2} \\
& \times\left[\left(v-\frac{1}{2}\right)\left(\rho_{1}+\rho_{2}\right)-\frac{1}{2}-\sqrt{\rho_{1}-\rho_{2}}\right]
\end{aligned}
$$

and we again used some algebraic factorization in the $a, b$ variables. From (11), $Z_{+}(w)=\left[\rho_{1}+\rho_{2}+1+\right.$ $\left.2 \sqrt{\rho_{1} \rho_{2}} \sqrt{w_{b}-w}\right] /\left(2 \rho_{1}\right)$ and hence

$$
\begin{aligned}
& {\left[Z_{+}(w)\right]^{m-n}} \\
& =\left(\frac{\rho_{1}+\rho_{2}+1}{2 \rho_{1}}\right)^{m-n} \\
& \quad \times\left[1+\frac{2 \sqrt{\rho_{1} \rho_{2}}}{\left(\rho_{1}+\rho_{2}\right)+1}(m-n) \sqrt{w_{b}-w}\right. \\
& \left.\quad+O\left(w-w_{b}\right)\right] .
\end{aligned}
$$

By multiplying (146) by (148) and using (139), the leading term for $p(m, n)$ becomes

$$
\begin{aligned}
p(m, n) \sim & \left(1-\rho_{1}-\rho_{2}\right) \\
& \times \sqrt{\frac{w_{b}}{\pi}} n^{-3 / 2} w_{b}^{-n}\left(\frac{2 \rho_{1}}{\rho_{1}+\rho_{2}+1}\right)^{m-n} \\
& \times \frac{A_{f}}{2 C}\left[\frac{2 \sqrt{\rho_{1} \rho_{2}}}{\rho_{1}+\rho_{2}+1}(m-n)+\frac{D}{C}\right] .
\end{aligned}
$$


But (149) is the same as (48), with $\bar{C}_{+}=\left(1-\rho_{1}-\rho_{2}\right) A_{f} /(2 C)$ and $\Delta_{+}=D / C$, since $D$ in (137) may also be written as

$$
D=-2 \sqrt{\rho_{1} \rho_{2}}\left[\rho_{2}(1-\nu)-\rho_{1}+\frac{1}{4}\left(\rho_{1}+\rho_{2}+1\right)^{2}\right] .
$$

We have thus established (48).

Now consider $R \in[0,1)$ with $\left(\rho_{1}+\rho_{2}\right)^{2}<\rho_{1}-\rho_{2}$. The integrand in (15) again has a saddle at $w_{s}^{*}(R)$, and $w_{s}^{*} \rightarrow 0$ as $R \rightarrow 0$. For $m=O(1)$ and $n \rightarrow \infty$ we again obtain (46). As $R \uparrow 1$ the saddle $w_{s}^{*}(R) \rightarrow w_{s}^{*}(1)=w_{b}^{*}>w_{b}$. Thus there is a critical value of $R$, which we call $R_{d}$, such that

$$
w_{s}^{*}\left(R_{d}\right)=w_{b} \text {. }
$$

Using (130), and the facts that $F_{*}^{\prime}\left(w_{s}\right)=0$ and $\sqrt{\left(\rho_{1}+\rho_{2}+1\right)^{2}-4 \rho_{2}\left(\rho_{1} w_{b}+1\right)}=\sqrt{\rho_{1}-\rho_{2}}$, we obtain

$$
\frac{R_{d}}{w_{b}}\left[\rho_{1}+\rho_{2}+1+2 \sqrt{\rho_{1}-\rho_{2}}\right]=\frac{\rho_{1} \rho_{2}}{\sqrt{\rho_{1}-\rho_{2}}}\left(1-R_{d}\right),
$$

which is a linear equation for $R_{d}$ whose solution is given by (54). If $0<R<R_{d}$ then $w_{s}^{*}(R)<w_{b}$ and the saddle point approximation in (42) holds. If $R_{d}<R<1$ the branch point at $w=w_{b}$ determines the asymptotics of $p(m, n)$, and we again use singularity analysis. Now we expand $g(w)$ about $w=w_{b}$, writing (17) as

$$
\operatorname{num}_{g}(w)=A_{g}+B_{g} \sqrt{w_{b}-w}+O\left(w-w_{b}\right),
$$

where

$$
\begin{gathered}
A_{g}=-2 \rho_{1} \rho_{2} w_{b}+\rho_{2}^{2}(1-\nu)+\rho_{1} \rho_{2}+\rho_{2}(1-v) \\
+\left(\rho_{1}^{2}-\rho_{1}\right) \nu, \\
B_{g}=\left[\left(\rho_{1}+\rho_{2}\right) \nu-\rho_{2}\right] 2 \sqrt{\rho_{1} \rho_{2} .}
\end{gathered}
$$

If we furthermore scale $w-w_{b}=U / n=O\left(n^{-1}\right)$, then

$$
\begin{aligned}
{\left[Z_{+}^{*}(w)\right]^{m-n} } & =\left[Z_{+}^{*}\left(w_{b}\right)+\frac{U}{n} Z_{+}^{* \prime}\left(w_{b}\right)+O\left(n^{-2}\right)\right]^{m-n} \\
& \sim\left[Z_{+}^{*}\left(w_{b}\right)\right]^{m-n} \exp \left[(R-1) \frac{Z_{+}^{* \prime}\left(w_{b}\right)}{Z_{+}^{*}\left(w_{b}\right)} U\right] \\
& w^{-m-1} \sim w_{b}^{-m-1} \exp \left(-\frac{R U}{w_{b}}\right) \\
g(w)= & \frac{A_{g}}{C}+\frac{B_{g} C-A_{g} D}{C^{2}} \frac{\sqrt{-U}}{\sqrt{n}}+O\left(\frac{1}{n}\right)
\end{aligned}
$$

It follows, by using (155)-(157) in (15), that

$p(m, n)$

$$
\begin{aligned}
& \sim\left(1-\rho_{1}-\rho_{2}\right) \frac{B_{g} C-A_{g} D}{C^{2}} n^{-3 / 2} w_{b}^{-m-1}\left[Z_{+}^{*}\left(w_{b}\right)\right]^{m-n} \\
& \quad \times \frac{1}{2 \pi i} \int_{\mathrm{Br}_{-}} \exp \left\{\left[-\frac{R}{w_{b}}+(R-1) \frac{Z_{+}^{* \prime}\left(w_{b}\right)}{Z_{+}^{*}\left(w_{b}\right)}\right] U\right\} \\
& \quad \times \sqrt{-U} d U,
\end{aligned}
$$

where $\operatorname{Re}(U)<0$ on $\mathrm{Br}_{-}$. After some calculation we find that, again using $a=\rho_{1}+\rho_{2}$ and $b=\sqrt{\rho_{1}-\rho_{2}}$,

$$
\begin{aligned}
A_{g}=(b+1) & (b-1)\left[\frac{1}{2}+\left(v-\frac{1}{2}\right) a\right]<0 \\
B_{g} C-A_{g} D= & -\frac{1}{4} \sqrt{\rho_{1} \rho_{2}}(1-b)^{2}(-2 v a+1+a+2 b) \\
& \times\left[2 \nu\left(b^{2}+a\right)+a^{2}+1-2 b^{2}\right] \\
= & -2 \sqrt{\rho_{1} \rho_{2}}\left(1-\sqrt{\rho_{1}-\rho_{2}}\right)^{2} \\
& \times\left[\frac{1}{2}+\sqrt{\rho_{1}-\rho_{2}}+\left(\rho_{1}+\rho_{2}\right)\left(\frac{1}{2}-v\right)\right] \\
& \times\left[\frac{1}{4}\left(\rho_{1}+\rho_{2}+1\right)^{2}-\rho_{1}(1-v)\right] \\
-\frac{R}{w_{b}}+ & (R-1) \frac{Z_{+}^{* \prime}\left(w_{b}\right)}{Z_{+}^{*}\left(w_{b}\right)}=-\frac{1}{w_{b}} \frac{R-R_{d}}{1-R_{d}} .
\end{aligned}
$$

The integral in (158) can then be evaluated as

$$
\begin{gathered}
\frac{1}{2 \pi i} \int_{\mathrm{Br}_{-}} \sqrt{-U} \exp \left[-\frac{1}{w_{b}} \frac{R-R_{d}}{1-R_{d}} U\right] d U \\
=w_{b}^{3 / 2}\left(\frac{1-R_{d}}{R-R_{d}}\right)^{3 / 2}\left(\frac{-1}{2 \sqrt{\pi}}\right) .
\end{gathered}
$$

Combining (141) with (158)-(162) we obtain precisely the result in (54)-(56).

Now consider the case $R \approx R_{d}$, where the branch point at $w=w_{b}$ is close to the saddle at $w=w_{s}^{*}(R)$. The standard scaling for such situations with coalescing singular points (see [18]) is $w-w_{b}=O\left(n^{-1 / 2}\right)$ and $R-R_{d}=O\left(n^{-1 / 2}\right)$. By expanding in Taylor series about $w=w_{b}$, setting $\left(R-R_{d}\right) \sqrt{n}=$ $S$ and scaling $S$ as in (59), and letting

$$
w-w_{b}=\frac{w_{b}}{\sqrt{n}}\left\{R_{d}\left[\frac{1}{1-R_{d}}+\frac{\rho_{1} \rho_{2} w_{b}}{2\left(\rho_{1}-\rho_{2}\right)}\right]\right\}^{-1 / 2} V
$$

we find that

$$
\begin{aligned}
\exp \{- & \left.m \log w+(m-n) \log \left[Z_{+}^{*}(w)\right]\right\} \\
= & w_{b}^{-m}\left[Z_{+}^{*}\left(w_{b}\right)\right]^{m-n} \exp \left[-S_{1} V+\frac{1}{2} V^{2}\right] \\
& \times\left[1+O\left(n^{-1 / 2}\right)\right] .
\end{aligned}
$$


Using (163) and (164) in (15) and again expanding $g(w)$ as in (157) we obtain

$$
\begin{aligned}
& p(m, n) \\
& =\left(1-\rho_{1}-\rho_{2}\right) M_{d}^{-1 / 2} w_{b}^{-m}\left[Z_{+}^{*}\left(w_{b}\right)\right]^{m-n} n^{-1 / 2} \\
& \times \frac{1}{2 \pi i} \int_{\mathrm{Br}_{-}} e^{-S_{1} V} e^{V^{2} / 2} \\
& \times\left[\frac{A_{g}}{C}+\frac{B_{g} C-A_{g} D}{C^{2}} \sqrt{w_{b}} M_{d}^{-1 / 4} \frac{\sqrt{-V}}{n^{1 / 4}}\right. \\
& \left.\quad+O\left(n^{-1 / 2}\right)\right] d V
\end{aligned}
$$

where $\mathrm{Br}_{-}$is to the left of all singularities. Here we also used the definition of $M_{d}$ in (58). The integral(s) in (165) may be easily evaluated using

$$
\begin{gathered}
\frac{1}{2 \pi i} \int_{\mathrm{Br}_{-}} e^{-S_{1} V} e^{V^{2} / 2} d V=\frac{e^{-S_{1}^{2} / 2}}{\sqrt{2 \pi}}, \\
\frac{1}{2 \pi i} \int_{\mathrm{Br}_{-}} e^{-S_{1} V} e^{V^{2} / 2} \sqrt{-V} d V=\frac{e^{-S_{1}^{2} / 4}}{\sqrt{2 \pi}} D_{1 / 2}\left(-S_{1}\right),
\end{gathered}
$$

where the latter follows from (61), after replacing $-V$ by $V$. But $g\left(w_{b}\right)=A_{g} / C$ by (157), and then (165) with (166) give precisely the approximation in (57)-(60). Note that $K$ is the same as $\left(B_{g} C-A_{g} D\right) / C^{2}$.

We can easily compute higher order terms in the expansion, and our analysis shows that the asymptotic series will now involve powers of $n^{-1 / 4}$. Actually, the leading term in (57), which has a Gaussian dependence on $S_{1}$ (hence on $S)$, can be obtained by simply expanding the saddle point approximation in (42), for $R \uparrow R_{d}$. However, the $O\left(n^{-1 / 4}\right)$ correction term is necessary to see the transition to the range $R>R_{d}$, where (55) applies. In view of (62) the correction term becomes comparable to the leading term when

$$
e^{-S_{1}^{2} / 2} \sim n^{-1 / 4} S_{1}^{-3 / 2}, \quad S_{1}>0
$$

or, roughly, when $\left|S_{1}\right| \sim \sqrt{(1 / 2) \log n}$. Thus for $S_{1}=O(1)$ or $S_{1} \gg 1$ with $\left|S_{1}\right| \ll \sqrt{(1 / 2) \log n}$ the Gaussian dominates while for $\left|S_{1}\right| \gg \sqrt{(1 / 2) \log n}$ the $O\left(n^{-1 / 4}\right)$ term dominates. Note that $K>0$ and $D_{1 / 2}\left(-S_{1}\right)<0$ for $S_{1}$ sufficiently negative, by (62). Thus for $S_{1} \sim \sqrt{(1 / 2) \log n}$ both the $O(1)$ and $O\left(n^{-1 / 4}\right)$ terms in (57) are positive. The asymptotic matching of (57) for $S_{1} \rightarrow+\infty$ (with $S_{1} \gg \sqrt{(1 / 2) \log n}$ ) with (55) as $R \downarrow R_{d}$ is then easily verified, as the approximation in (55) has an $O\left[\left(R-R_{d}\right)^{-3 / 2}\right]$ algebraic singularity as $R \downarrow$ $R_{d}$, which agrees with the second formula in (62). Also, up to a multiplicative constant $n^{-3 / 2}\left(R-R_{d}\right)^{-3 / 2}$ agrees with $N^{-3 / 4} S_{1}^{-3 / 2}$. This completes the derivation of Theorem 3 , where $\left(\rho_{1}+\rho_{2}\right)^{2}<\rho_{1}-\rho_{2}$.
Next we analyze how the results in Theorem 2 transition to those in Theorem 3 , as $\left(\rho_{1}+\rho_{2}\right)^{2}$ decreases through $\rho_{1}-$ $\rho_{2}(>0)$. We could simply assume that $\left(\rho_{1}+\rho_{2}\right)^{2}=\rho_{1}-\rho_{2}$ and then obtain the necessary asymptotic results. But to see the transition it is necessary to also analyze cases where $\left(\rho_{1}+\right.$ $\left.\rho_{2}\right)^{2} \approx \rho_{1}-\rho_{2}$. To make this more precise we write

$$
\left(\rho_{1}+\rho_{2}\right)^{2}-\left(\rho_{1}-\rho_{2}\right)=\delta \beta,
$$

as in (63), and assume that $\delta \rightarrow 0^{+}$. Then $\beta \gtrless 0$ according as $\left(\rho_{1}+\rho_{2}\right)^{2} \gtrless \rho_{1}-\rho_{2}$. Since only the product $\delta \beta$ is important, we can set $\beta=+1,0,-1$, according to the cases $\left(\rho_{1}+\rho_{2}\right)^{2}>$ $\rho_{1}-\rho_{2},\left(\rho_{1}+\rho_{2}\right)^{2}=\rho_{1}-\rho_{2},\left(\rho_{1}+\rho_{2}\right)^{2}<\rho_{1}-\rho_{2}$. Then we must relate the small parameter $\delta$ to the large parameters $m, n$, and we show below that a natural scaling is to take $m, n=O\left(\delta^{-2}\right)$ as $\delta \rightarrow 0^{+}$.

The asymptotic results for $m \rightarrow \infty$ and $n=O(1)$ are the same in Theorems 2 and 3 , and thus no transition is needed here. We can write these results in terms of, say, $\left(\rho_{1}, \delta \beta\right)$ rather than $\left(\rho_{1}, \rho_{2}\right)$ and expand for small $\delta$ to somewhat simplify the expression in (25), but we will not do so here. If $\left(\rho_{1}+\rho_{2}\right)^{2}<$ $\rho_{1}-\rho_{2}$ the saddle point approximation in (27) applies for all $R>1$, while if $\left(\rho_{1}+\rho_{2}\right)^{2}>\rho_{1}-\rho_{2}$ it applies only for $R>R_{c}$. But $R_{c} \rightarrow 1$ (cf. (26)) as $\left(\rho_{1}+\rho_{2}\right)^{2} \rightarrow \rho_{1}-\rho_{2}$ so it will apply for any fixed $R>1$. But $R \gtrsim 1$ will require a separate analysis. We also note that the sector $1<R<R_{c}$, where the product form solution in (34) applies, shrinks to zero. Thus if such an approximation will play a role here, it must be contained near $R=1$.

We begin by considering the diagonal probabilities $p(n, n)$, using the scaling in (168). We will approximate $h(w)$ in (19) in such a way that the integrand approaches a nondegenerate limit. The pole at $w_{p}=\left(\rho_{1}+\rho_{2}\right)^{-2}$, if it exists, is now close to the branch point $w_{b}=\left[\left(\rho_{1}+\rho_{2}+\right.\right.$ $\left.1)^{2}-4 \rho_{1}\right] /\left(4 \rho_{1} \rho_{2}\right)$, since $\left(\rho_{1}+\rho_{2}\right)^{2} \approx \rho_{1}-\rho_{2}$. Note that $a^{-2}=\left[(a+1)^{2}-2\left(a+b^{2}\right)\right] /\left(a^{2}-b^{4}\right)$ if $a\left(=\rho_{1}+\rho_{2}\right)=$ $b\left(=\sqrt{\rho_{1}-\rho_{2}}\right)$. Introducing the scaling $w=w_{b}(1+U / n)$, so that $w-w_{b}=O\left(n^{-1}\right)$, we have $w^{-n-1} d w \sim n^{-1} w_{b}^{-n} e^{-U} d U$. Now we write $h(w)$ near $w_{b}$ as

$$
h(w)=\frac{A+O\left(\sqrt{w_{b}-w}\right)}{C+D \sqrt{w_{b}-w}+O\left(w-w_{b}\right)} .
$$

From (134) we find that

$$
A \longrightarrow-\frac{1}{2}(1-a)^{2}(1+a) \text { as } b \longrightarrow a\left(\text { or } \delta \longrightarrow 0^{+}\right),
$$

while (136) or (141) shows that

$$
\begin{aligned}
C & \sim(b-a)(1-a)\left(\frac{3}{2} a+\frac{1}{2}-a v\right) \\
& \sim-\frac{\delta \beta}{4 a}(1-a)(3 a+1-2 a v),
\end{aligned}
$$

since $a^{2}-b^{2}=\delta \beta$ and thus $a-b=\delta \beta /(a+b) \sim \delta \beta /(2 a)$. From (137) or (151) we also find that as $b \rightarrow a$

$$
D \longrightarrow-\frac{1}{4} a(1-a) \sqrt{1-a^{2}}(3 a+1-2 a v) \text {. }
$$


Then from (169)-(172) we see that $C$ becomes comparable to $D \sqrt{w_{b}-w}$ if $\delta=O\left(n^{-1 / 2}\right)$, or $n=O\left(\delta^{-2}\right)$. Then setting $n=$ $y / \delta^{2}$ and using (169)-(172), we can approximate (19) to obtain

$$
\begin{aligned}
p(m, n) \sim & \frac{(1-a)^{2}}{\delta n} \frac{2(1+a)}{3 a+1-2 a \nu} w_{b}^{-n} \\
& \times \frac{1}{2 \pi i} \int_{\mathrm{Br}_{-}} \frac{e^{-U}}{-(\beta / a)+\sqrt{1-a^{2}}(\sqrt{-U} / \sqrt{y})} d U .
\end{aligned}
$$

Thus it is useful to introduce $\beta_{1}=\beta /\left(a \sqrt{1-a^{2}}\right)$ and then we evaluate the contour integral in (173) as, changing $U \rightarrow-U$,

$$
\begin{aligned}
& \frac{1}{2 \pi i} \int_{\mathrm{Br}_{+}} \frac{e^{U} \sqrt{y}}{\sqrt{U}-\beta_{1} \sqrt{y}} d U \\
& =\frac{2 \sqrt{y}}{2 \pi i} \int_{\mathrm{Br}_{+}} e^{Z^{2}} \frac{\left(Z-\beta_{1} \sqrt{y}+\beta_{1} \sqrt{y}\right)}{Z-\beta_{1} \sqrt{y}} d Z \\
& =\frac{\sqrt{y}}{\sqrt{\pi}}+\frac{\sqrt{y}}{\pi i} \beta_{1} \sqrt{y} \int_{\mathrm{Br}_{+}} \frac{e^{Z^{2}}}{Z-\beta_{1} \sqrt{y}} d Z \\
& =\sqrt{\frac{y}{\pi}}\left[1+\frac{\beta_{1} \sqrt{y}}{\sqrt{\pi} i} e^{\beta_{1}^{2} y} \int_{\mathrm{Br}_{+}} \frac{e^{V^{2}} e^{2 \beta_{1} \sqrt{y} V}}{V} d V\right] \\
& =\sqrt{\frac{y}{\pi}}\left[1+2 \beta_{1} \sqrt{y} e^{\beta_{1}^{2} y} \int_{-\infty}^{\beta_{1} \sqrt{y}} e^{-u^{2}} d u\right] .
\end{aligned}
$$

Here we used a conformal map $U=Z^{2}$, some contour deformations, (61) with $p=-1$, and an identity that relates $D_{-1}(\cdot)$ to the standard error function, which yields the last equality in (174). Using (174) in (173) and noting that $\sqrt{y} /(\delta n)=n^{-1 / 2}$, we obtain (66).

Now consider $R \gtrsim 1$ and we already discussed the case $R>1$ with the transition range scaling in (168). We expand now (10) similarly as we expanded (15). For $w-w_{b}=O\left(n^{-1}\right)$, the factor $\left[Z_{+}(w)\right]^{n-m}$ can be approximated by

$$
\begin{aligned}
& {\left[Z_{+}(w)\right]^{n-m}} \\
& \quad \sim\left(\frac{2 \rho_{1}}{\rho_{1}+\rho_{2}+1}\right)^{m-n} \exp \left[\frac{2 \sqrt{\rho_{1} \rho_{2} w_{b}}}{\rho_{1}+\rho_{2}+1} \frac{\sqrt{-U}}{\sqrt{n}}(n-m)\right]
\end{aligned}
$$

for $m-n=O(\sqrt{n})$. We thus let $z=\delta(m-n)$ so that $(m-$ $n) / \sqrt{n}=z / \sqrt{y}$. Also, $w_{b} \rightarrow a^{-2}$ as $\delta \rightarrow 0^{+}$, and then

$$
\frac{2 \sqrt{\rho_{1} \rho_{2} w_{b}}}{\rho_{1}+\rho_{2}+1} \longrightarrow \sqrt{\frac{1-a}{1+b}}, \quad b \longrightarrow a
$$

Hence (10) becomes

$$
\begin{aligned}
p(m, n) \sim & \left(1-\rho_{1}-\rho_{2}\right) w_{b}^{-n}\left(\frac{2 \rho_{1}}{\rho_{1}+\rho_{2}+1}\right)^{m-n} \frac{1}{n} \\
& \times \frac{1}{2 \pi i} \int_{\text {Br- }_{-}} \frac{A_{f}}{C+D \sqrt{w_{b}} \sqrt{-U / n}} e^{-U} \\
& \times \exp \left[-\sqrt{\frac{1-a}{1+a}} \sqrt{-U} \frac{Z}{\sqrt{y}}\right] d U .
\end{aligned}
$$

Now $C$ and $D$ can be evaluated as in (171) and (172), and, as $b \rightarrow a, A_{f}$ in (147) becomes

$$
A_{f} \sim-\frac{1}{2}(1-a)^{2}(3 a+1-2 v a) .
$$

Then (177) can be evaluated similarly to (173), and we ultimately obtain (68), with $\widetilde{z}=\sqrt{(1-a) /(1+a)} z \sqrt{y}$ as in (69).

For $R<1(n>m)$ we need only consider the ranges $R \in\left(R_{d}^{0}, 1\right)$ and $R \approx R_{d}^{0}$, where $R_{d}^{0}$ is in (64), with $R_{d}^{0}$ being the limit of both $R_{c}^{*}$ and $R_{d}$, as $b \rightarrow a$ or $\delta \rightarrow 0^{+}$. For $R>R_{d}^{0}$ the saddle at $w_{s}^{*}(R)$ exceeds the branch point $w_{b}$ and hence the latter determines the asymptotic behavior of $p(m, n)$. For a fixed $R$, we scale $w=w_{b}+U / n$ and use

$$
\begin{gathered}
w^{-m-1} \sim w_{b}^{-m-1} \exp \left(-\frac{R}{w_{b}} U\right), \\
{\left[Z_{+}^{*}(w)\right]^{m-n} \sim\left[Z_{+}^{*}\left(w_{b}\right)\right]^{m-n} \exp \left[(R-1) \frac{Z_{+}^{* \prime}\left(w_{b}\right)}{Z_{+}^{*}\left(w_{b}\right)} U\right] .}
\end{gathered}
$$

We again expand $g(w)$ in the form in (169), with $A$ now replaced by $A_{g}$, where

$$
A_{g} \sim-\frac{1}{2}\left(1-a^{2}\right)(1-a+2 v a), \quad b \longrightarrow a .
$$

Using (161) with $R_{d}$ replaced by $R_{d}^{0}$, along with (180) and (181), the integral in (7) becomes

$$
\begin{aligned}
& \frac{(1-a)(1+a)(1-a+2 v a)}{3 a+1-2 v a} \frac{2}{n \delta} w_{b}^{-m-1}\left[Z_{+}^{*}\left(w_{b}\right)\right]^{m-n} \\
& \quad \times \frac{1}{2 \pi i} \int_{\mathrm{Br}_{+}} \frac{\exp \left(\left(V / w_{b}\right)\left(\left(R-R_{d}^{0}\right) /\left(1-R_{d}^{0}\right)\right)\right)}{-(\beta / a)+\sqrt{1-a^{2}} a(\sqrt{V} / \sqrt{y})} d V .
\end{aligned}
$$

Here we let $U=-V$ and used (171) and (172) to approximate $C$ and $D$. Scaling $\beta$ as in (178) and evaluating the integral in (182) similarly to (173) leads to (70). If $n-m=O(1)$, the same analysis applies, as then we can simply replace $R$ by 1 and $\beta_{2}$ by $\beta_{1}$ in (70) and (71), but must maintain the factor $\left[Z_{+}^{*}\left(w_{b}\right)\right]^{m-n}$.

When $R \approx R_{d}^{0}$ we let $S^{0}=\left(R-R_{d}^{0}\right) \sqrt{n}=O(1)$. Now the saddle $w_{s}^{*}(R)$ will be close to the branch point $w_{b}$ (with also $w_{b} \sim a^{-2}$ by (168)). We scale $w-w_{b}=O\left(n^{-1 / 2}\right)$ with

$$
w-w_{b}=\frac{-V}{\sqrt{n}} w_{b}\left\{R_{d}\left[\frac{1}{1-R_{d}}+\frac{\rho_{1} \rho_{2} w_{b}}{2\left(\rho_{1}-\rho_{2}\right)}\right]\right\}^{-1 / 2}
$$


and introduce $S_{1}^{0}$ as in (73). For $b \sim a$ we also have

$$
\frac{1}{1-R_{d}}+\frac{\rho_{1} \rho_{2} w_{b}}{2\left(\rho_{1}-\rho_{2}\right)} \sim \frac{1+5 a+7 a^{2}+19 a^{3}}{4 a(1+3 a)}
$$

and then

$$
\frac{S_{1}^{0}}{S^{0}}=\frac{\left(1+4 a+11 a^{2}\right)^{3 / 2}}{2 \sqrt{a(1+3 a)}} \frac{1}{\sqrt{1-a^{2}}} \frac{1}{\sqrt{1+5 a+7 a^{2}+19 a^{3}}} .
$$

Since $C=O(\delta)$ and $D \sqrt{w_{b}-w}=O\left(n^{-1 / 4}\right)=O(\sqrt{\delta})$ we have

$$
\begin{aligned}
g(w) & =\frac{A_{g}+O\left(w_{b}-w\right)}{C+D \sqrt{w_{b}-w}+O\left(w_{b}-w\right)} \\
& \sim \frac{A_{g}}{D} \frac{1}{\sqrt{w_{b}-w}} \sim \frac{2}{a} \sqrt{\frac{1+a}{1-a}} \frac{1-a+2 v a}{1+3 a-2 v a} \frac{1}{\sqrt{w_{b}-w}}
\end{aligned}
$$

and then

$$
\begin{aligned}
p(m, n) \sim & \frac{A_{g}}{D}(1-a) \frac{1}{n^{1 / 4} \sqrt{w_{b}}}\left[R_{d}^{0}\left(\frac{1}{1-R_{d}^{0}}+\frac{1-a^{2}}{8 a^{2}}\right)\right]^{-1 / 4} \\
& \times w_{b}^{-m}\left[Z_{+}^{*}\left(w_{b}\right)\right]^{m-n} \frac{1}{2 \pi i} \int_{\mathrm{Br}_{+}} \frac{e^{S_{1}^{0} V} e^{V^{2} / 2}}{\sqrt{V}} d V .
\end{aligned}
$$

Expressing the integral as a parabolic cylinder function of order $p=-1 / 2$ (see (61)) we obtain the expression in (72), with (73) or (185).

If we let $\delta \rightarrow 0^{+}$but consider even larger values of $n$, with $n=y_{1} / \delta^{4}=O\left(\delta^{-4}\right)$, a slightly different expansion applies. Now both $C$ and $\sqrt{w_{b}-w}$ in (186) become $O(\delta)$. Setting $w-$ $w_{b}=-\bar{V} / \sqrt{n}$, we use

$$
g(w) \sim \frac{2(1+a)(1-a+2 v a)}{3 a+1-2 v a}\left[\frac{-\beta y_{1}^{1 / 4}}{a}+a \sqrt{1-a^{2}} \sqrt{\bar{V}}\right]^{-1}
$$

in (15) to ultimately obtain the expression in (74), which involves the contour integral in (76). The function $\mathscr{L}(Z ; \gamma)$ can be expressed as an infinite sum of parabolic cylinder functions, as

$$
\mathscr{L}(Z ; \gamma)=\left[\sum_{j=0}^{\infty} \gamma^{j} D_{-(j+1) / 2}(-Z)\right] \frac{e^{-Z^{2} / 4}}{\sqrt{2 \pi}} .
$$

This completes the analysis of the transition range where $\left(\rho_{1}+\rho_{2}\right)^{2} \approx \rho_{1}-\rho_{2}$, and we have thus established Theorem 4 .

\section{Light and Heavy Traffic}

We establish Theorem 5. First consider the light traffic limit, where $\rho_{1}, \rho_{1} \rightarrow 0$. We now scale

$$
w=\frac{u}{\rho_{1} \rho_{2}}
$$

and then, from (11), (12), (16), and (20), obtain

$$
\begin{gathered}
Z_{+}(w) \sim \frac{1}{2 \rho_{1}}[1+\sqrt{1-4 u}], \\
Z_{+}^{*}(w) \sim \frac{1}{2 \rho_{2}}[1+\sqrt{1-4 u}], \\
f(w) \sim g(w) \sim h(w) \sim \frac{1}{\sqrt{1-4 u}} .
\end{gathered}
$$

Hence the diagonal probabilities have the limiting form

$$
\begin{aligned}
p(n, n) & \sim\left(\rho_{1} \rho_{2}\right)^{n} \frac{1}{2 \pi i} \oint \frac{u^{-n-1}}{\sqrt{1-4 u}} d u \\
& =\left(\rho_{1} \rho_{2}\right)^{n}\left[u^{n}\right](1-4 u)^{-1 / 2} \\
& =\rho_{1}^{n} \rho_{2}^{n}\left(\begin{array}{c}
2 n \\
n
\end{array}\right),
\end{aligned}
$$

where we used the binomial theorem, and $\left[u^{n}\right]$ denotes the coefficient of $u^{n}$ in the Taylor expansion of what follows.

For $m>n$ we similarly obtain

$$
\begin{aligned}
p(m, n) \sim & \left(\rho_{1} \rho_{2}\right)^{n}\left(2 \rho_{1}\right)^{m-n} \\
& \times \frac{1}{2 \pi i} \oint \frac{1}{u^{n+1}}\left(\frac{1}{1+\sqrt{1-4 u}}\right)^{m-n} \frac{d u}{\sqrt{1-4 u}} \\
= & \rho_{1}^{m} \rho_{2}^{n}\left(\begin{array}{c}
m+n \\
n
\end{array}\right),
\end{aligned}
$$

and this holds also for $m \leqslant n$. By refining (191) with (190) we can obtain higher order terms, as an expansion involving powers of $\rho_{1}$ and $\rho_{2}$. We have thus established (78).

For the first heavy traffic limit (HTL1) we let $\varepsilon=1-\rho_{1}-$ $\rho_{2} \rightarrow 0^{+}$, replace $\rho_{2}$ by $1-\rho_{1}-\varepsilon$ and also set

$$
n=\frac{Y}{\varepsilon}, \quad m=n+\ell, \quad w=1-\varepsilon \xi .
$$

Then after some calculation we find from (14) that

$$
\operatorname{den}(w)=\frac{1}{4} \varepsilon^{2} \xi(2+\xi)\left(2 v \rho_{2}-v+2-\rho_{2}\right)+O\left(\varepsilon^{3}\right),
$$

and from (21), (13), and (17) that

$$
\begin{gathered}
\operatorname{num}(w)=\varepsilon \xi \rho_{2}\left(1-\rho_{2}\right)+O\left(\varepsilon^{2}\right), \\
\operatorname{num}_{f}(w)=\varepsilon \xi \rho_{2}\left(2-\nu-\rho_{2}\right)+O\left(\varepsilon^{2}\right), \\
\operatorname{num}_{g}(w)=\varepsilon \xi\left(1-\rho_{2}\right)\left(\nu+\rho_{2}\right)+O\left(\varepsilon^{2}\right) .
\end{gathered}
$$

Hence the limiting form of (19) is

$$
\begin{aligned}
p(n, n) & \sim \varepsilon \frac{4 \rho_{2}\left(1-\rho_{2}\right)}{2 \nu \rho_{2}-v+2 \rho_{2}} \frac{1}{2 \pi i} \int_{\mathrm{Br}_{+}} \frac{e^{Y \xi}}{\xi+2} d \xi \\
& =\varepsilon \frac{4 \rho_{2}\left(1-\rho_{2}\right)}{2 \nu \rho_{2}-v+2-\rho_{2}} e^{-2 Y},
\end{aligned}
$$


which is the same as (80). With the scaling $w=1-\varepsilon \xi$ the loop integral in (19) can be approximated by the vertical contour $\mathrm{Br}_{+}$, on which $\operatorname{Re}(\xi)>-2$ in this case. Since $w^{-n-1} \sim w^{-m-1} \sim$ $e^{Y \xi}$ and

$$
Z_{+}(w) \sim \frac{1+\rho_{2}}{1-\rho_{2}}, \quad Z_{+}^{*}(w) \sim \frac{2-\rho_{2}}{\rho_{2}}
$$

we use (200), (195), (197), and (198) to approximate the integrands in (10) and (15), and this leads to (79) and (81).

We can also derive the HTL1 limits directly from the asymptotic formulas in (34), (35), and (38), as for $\rho_{1}=1-\rho_{2}-\varepsilon$

$$
\begin{aligned}
& C_{0} \sim \frac{4 \rho_{2}\left(1-\rho_{2}\right)}{2-\rho_{2}+\left(2 \rho_{2}-1\right) \nu} \varepsilon, \\
& C_{+} \sim \frac{4 \rho_{2}\left(2-\nu-\rho_{2}\right)}{2-\rho_{2}+\left(2 \rho_{2}-1\right) \nu} \varepsilon, \\
& C_{-} \sim \frac{4\left(1-\rho_{2}\right)\left(\rho_{2}+\nu\right)}{2-\rho_{2}+\left(2 \rho_{2}-1\right) \nu} \varepsilon
\end{aligned}
$$

and $\left(\rho_{1}+\rho_{2}\right)^{2 n}=(1-\varepsilon)^{2 Y / \varepsilon} \sim e^{-2 Y}$. This shows that it is permissible in this case to first let $m, n \rightarrow \infty$ with a fixed $\rho_{1}, \rho_{2}$ and then let $\rho_{1}+\rho_{2} \uparrow 1$. Note also that in HTL1 the condition $\left(\rho_{1}+\rho_{2}\right)^{2}>\rho_{1}-\rho_{2}$ is certainly satisfied, so Theorem 2 applies.

In the second heavy traffic limit, HTL2, we again let $\rho_{1}+$ $\rho_{2}=1-\varepsilon$ and $w=1-\varepsilon \xi$, but now $\rho_{2}=\gamma \varepsilon=O(\varepsilon)$ is small. We now set $m=X / \varepsilon$ and $n=Y / \varepsilon$, and we have

$$
\begin{gathered}
\left(1+\rho_{1}+\rho_{2}\right)^{2}-4 \rho_{1}-4 \rho_{1} \rho_{2} w \\
=\varepsilon^{2}\left[(1+2 \gamma)^{2}+4 \gamma \xi\right]+O\left(\varepsilon^{3}\right) \\
Z_{+}(w)=1+\frac{1}{2}\left[2 \gamma+1+\sqrt{(2 \gamma+1)^{2}+4 \gamma \xi}\right] \varepsilon+O\left(\varepsilon^{2}\right) .
\end{gathered}
$$

Then after some calculation we find that as $\varepsilon \rightarrow 0$

$$
\begin{aligned}
h(w) & \longrightarrow h_{0}(\xi) \\
= & \frac{(2-\nu) \xi+(2 \gamma+1)(1-\nu)-(1-\nu) \sqrt{(2 \gamma+1)^{2}+4 \gamma \xi}}{(2-\nu)\left[\xi+2 \gamma+1-\sqrt{(2 \gamma+1)^{2}+4 \gamma \xi}\right]}
\end{aligned}
$$

and thus $p(n, n)$ is $O\left(\varepsilon^{2}\right)$ and given by the expression in (84) (again we have $w^{-n-1} \sim e^{Y \xi}$ ). Since $h_{0}(\xi) \rightarrow 1$ as $\xi \rightarrow \infty$, the right side of (84) has probability mass along $Y=0$, and we can write

$$
\begin{aligned}
& \frac{1}{2 \pi i} \int_{\mathrm{Br}_{+}} e^{Y \xi} h_{0}(\xi) d \xi \\
& \quad=\delta(Y)+\frac{1}{2 \pi i} \int_{\mathrm{Br}_{+}} \frac{e^{Y \xi}\left[\sqrt{(2 \gamma+1)^{2}+4 \gamma \xi}-(2 \gamma+1)\right]}{\left(\xi+2 \gamma+1-\sqrt{(2 \gamma+1)^{2}+4 \gamma \xi}\right]} d \xi .
\end{aligned}
$$

The presence of the $\delta(Y)$ term in (205) indicates that different approximations are needed for $p(n, n)$ for $Y>0$ and for small $Y=\varepsilon n$, such as $n=O(1)$. But in HTL2 there is little mass along the diagonal $m=n$ as a whole.

In HTL2 we also obtain

$$
\begin{aligned}
& \operatorname{num}_{f}(w)=\varepsilon^{2}(2-\nu) \gamma \xi+O\left(\varepsilon^{3}\right) \\
& \operatorname{den}(w)= \varepsilon^{2} \gamma(2-\nu) \\
& \times\left[\xi+2 \gamma+1-\sqrt{(2 \gamma+1)^{2}+4 \gamma \xi}\right]+O\left(\varepsilon^{3}\right)
\end{aligned}
$$

so that $f(w) \rightarrow f_{0}(\xi)$, as in (83), and then $p(m, n)$ is given by (82). We also note that, in view of (203) and the scaling $(X, Y)=\varepsilon(m, n)$,

$$
\left[Z_{+}(w)\right]^{n-m} \longrightarrow \exp \left\{\frac{Y-X}{2}\left[2 \gamma+1+\sqrt{(2 \gamma+1)^{2}+4 \gamma \xi}\right]\right\}
$$

If $\mathscr{F}(X, Y)$ is the limit of $\varepsilon^{-2} p(m, n)$, for $m>n$, in HTL2, then by contour integration

$$
\begin{aligned}
\int_{0}^{\infty} \int_{Y}^{\infty} \mathscr{F}(X, Y) d X d Y \\
\quad \equiv \int_{0}^{\infty}\left[\frac{1}{2 \pi i} \int_{\mathrm{Br}_{+}} \frac{2 f_{0}(\xi) e^{Y \xi}}{2 \gamma+1+\sqrt{(2 \gamma+1)^{2}+4 \gamma \xi}} d \xi\right] d Y \\
\quad=\frac{1}{2 \pi i} \int_{\mathrm{Br}_{-}} \frac{2}{2 \gamma+1+\sqrt{(2 \gamma+1)^{2}+4 \gamma \xi}}\left(-\frac{1}{\xi}\right) f_{0}(\xi) d \xi \\
\quad=\frac{1}{2 \gamma+1} f_{0}(0)=1
\end{aligned}
$$

so that $\mathscr{F}$ is a proper two-dimensional density function, with support in the range $X>Y>0$. In (208), on $\mathrm{Br}_{-}, \operatorname{Re}(\xi) \epsilon$ $\left(-(2 \gamma+1)^{2} / 4 \gamma, 0\right)$.

For $m<n$ in HTL2 we have

$$
Z_{+}^{*}(w) \sim \frac{2-\rho_{2}}{\rho_{2}} \sim \frac{2}{\varepsilon \gamma}
$$

so that $\left[Z_{+}^{*}(w)\right]^{m-n}=O\left(\varepsilon^{n-m}\right)$. For $n-m=O(1)$ we also have $w^{-m-1} \sim e^{X \xi} \sim e^{Y \xi}$ and

$$
\operatorname{num}_{g}(w)=\varepsilon \nu\left[\sqrt{(2 \gamma+1)^{2}+4 \gamma \xi}-(2 \gamma+1)\right]+O\left(\varepsilon^{2}\right)
$$

Then $g(w) \sim \varepsilon^{-1} \bar{g}(\xi)$ as below (86). Thus for $n>m$ we have derived (86), and this completes the analysis of HTL2. Note that in HTL2 both $p(n, n)$ and $p(n-1, n)$ are $O\left(\varepsilon^{2}\right)$. 


\section{Appendix}

Here we study the roots of $\operatorname{den}(w)=0$ in (14). We have $\operatorname{den}(1)=0$ and

$$
\begin{aligned}
\operatorname{den}^{\prime}(1)= & -\frac{2 \rho_{1} \rho_{2}\left(1-\rho_{1}-\rho_{2}\right)}{\left(1-\rho_{2}+\rho_{1}\right)\left(1-\rho_{1}+\rho_{2}\right)} \\
& \times\left[1+\rho_{2} \nu+\rho_{1}(1-\nu)\right],
\end{aligned}
$$

so that $\operatorname{den}^{\prime}(1)<0$ under the stability condition $\rho_{1}+\rho_{2}<1$. Setting

$$
\begin{aligned}
& \Delta_{1}=\left(\rho_{1}+\rho_{2}+1\right)^{2}-4 \rho_{1}\left(\rho_{2} w+1\right), \\
& \Delta_{2}=\left(\rho_{1}+\rho_{2}+1\right)^{2}-4 \rho_{2}\left(\rho_{1} w+1\right)
\end{aligned}
$$

we have

$$
\begin{aligned}
& \operatorname{den}^{\prime}(w)=-\rho_{1} \rho_{2}\left(\sqrt{\Delta_{1}}+\sqrt{\Delta_{2}}\right) \\
&+2 \rho_{1} \rho_{2}\left[\frac{\rho_{1}\left(\rho_{2} w+\nu\right)}{\sqrt{\Delta_{2}}}+\frac{\rho_{2}\left(\rho_{1} w+1-v\right)}{\sqrt{\Delta_{1}}}\right], \\
& \operatorname{den}^{\prime \prime}(w)=4 \rho_{1}^{2} \rho_{2}^{2}\left[\frac{1}{\sqrt{\Delta_{1}}}+\frac{1}{\sqrt{\Delta_{2}}}+\frac{\rho_{1}\left(\rho_{2} w+\nu\right)}{\Delta_{2}^{3 / 2}}\right. \\
&\left.+\frac{\rho_{2}\left(\rho_{1} w+1-\nu\right)}{\Delta_{1}^{3 / 2}}\right] .
\end{aligned}
$$

Now $\Delta_{1}=0$ when $w=w_{b}$ and $\Delta_{2}=0$ when $w=w_{b}^{*}>w_{b}$ (since $\rho_{1}>\rho_{2}$ ). We thus have $\operatorname{den}(w)$ real for $w$ real and $w \in$ $\left(-\infty, w_{b}\right)$. From (A.4) we conclude that $\operatorname{den}(w)$ is a convex function of $w$ for $w \in\left(-\infty, w_{b}\right)$. This is obvious for $w \geqslant 0$ and for $w<0$ we note that $\Delta_{1}+\rho_{2}\left(\rho_{1} w+1-v\right)>0$ and $\Delta_{2}+\rho_{1}\left(\rho_{2} w+\nu\right)>0$, in view of (A.2). Also, (A.3) shows that $\operatorname{den}^{\prime}\left(w_{b}\right)=+\infty$ and $\operatorname{den}^{\prime}(-\infty)=-\infty$. In view of the convexity and the fact that $\operatorname{den}(1)=0$ and $\operatorname{den}^{\prime}(1)<0$ we conclude that $\operatorname{den}(w)=0$ has at most two real roots. But as we discussed in Section 4, $w=w_{p}=\left(\rho_{1}+\rho_{2}\right)^{-2}>1$ is a root of $\operatorname{den}(w)=0$ if $\left(\rho_{1}+\rho_{2}\right)^{2}>\rho_{1}-\rho_{2}$. Setting $w=0$ yields

$$
\begin{array}{r}
\operatorname{den}(0)=\rho_{1} \nu\left[\rho_{1}+\rho_{2}+1-\sqrt{\left(\rho_{1}+\rho_{2}+1\right)^{2}-4 \rho_{2}}\right] \\
+\rho_{2}(1-\nu)\left[\rho_{1}+\rho_{2}+1\right. \\
\left.-\sqrt{\left(\rho_{1}+\rho_{2}+1\right)^{2}-4 \rho_{1}}\right]>0
\end{array}
$$

and setting $w=w_{b}$ leads to

$$
\begin{aligned}
\operatorname{den}\left(w_{b}\right)= & \left(\rho_{1}+\rho_{2}+1\right)\left[\rho_{1} \nu+\rho_{2}(1-\nu)\right] \\
& -2 \sqrt{\rho_{1}-\rho_{2}} \rho_{1}\left(\rho_{2} w_{b}+\nu\right) .
\end{aligned}
$$

To determine the sign of $\operatorname{den}\left(w_{b}\right)$ we use (49) and the variables $a=\rho_{1}+\rho_{2}$ and $b=\sqrt{\rho_{1}-\rho_{2}}$. Then $\operatorname{den}\left(w_{b}\right)>0$ is equivalent to

$$
(a+1)\left[a+(2 v-1) b^{2}\right]>\left[(a+1)^{2}+2(v-1)\left(a+b^{2}\right)\right] b
$$

which simplifies to

$$
(a-b)(1-b)[1+a+2(1-v) b]>0
$$

so that den $\left(w_{b}\right)>0$ according as $a>b$ or $\left(\rho_{1}+\rho_{2}\right)^{2}>\rho_{1}-\rho_{2}$, that is, when $w_{p}$ is a root. It follows that if $\left(\rho_{1}+\rho_{2}\right)^{2}>\rho_{1}-\rho_{2}$, $w=1$ and $w=w_{p}$ are two roots of $\operatorname{den}(w)=0$, and if $\left(\rho_{1}+\right.$ $\left.\rho_{2}\right)^{2}<\rho_{1}-\rho_{2}, w=1$ is the only root. Since $\operatorname{den}^{\prime}(1)<0$ and $\operatorname{den}^{\prime \prime}(w)>0$ there can be no roots for $w \leqslant 0$.

Now we consider the possibility of having complex roots of $\operatorname{den}(w)=0$. Writing

$$
\begin{aligned}
\operatorname{den}(w)= & \left(\rho_{1}+\rho_{2}+1\right)\left[\rho_{1} \nu+\rho_{2}(1-\nu)\right] \\
& -\rho_{1}\left(\rho_{2} w+\nu\right) \sqrt{\Delta_{2}}-\rho_{2}\left(\rho_{1} w+1-\nu\right) \sqrt{\Delta_{1}}
\end{aligned}
$$

we eliminate the radicals to obtain

$$
\begin{aligned}
& \left\{\left(\rho_{1}+\rho_{2}+1\right)^{2}\left[\rho_{1} \nu+\rho_{2}(1-v)\right]^{2}\right. \\
& \left.\quad-\rho_{1}^{2}\left(\rho_{2} w+\nu\right)^{2} \Delta_{2}-\rho_{2}^{2}\left(\rho_{1} w+1-\nu\right)^{2} \Delta_{1}\right\}^{2} \\
& =4 \rho_{1}^{2} \rho_{2}^{2}\left(\rho_{2} w+\nu\right)^{2}\left(\rho_{1} w+1-\nu\right)^{2} \Delta_{1} \Delta_{2} .
\end{aligned}
$$

Thus if $w$ is a root of $\operatorname{den}(w)$ it must be a root of (A.10). After some simplification and factoring using MAPLE, we find that solving (A.10) is equivalent to solving the quartic equation

$$
(w-1)\left[\left(\rho_{1}+\rho_{2}\right)^{2} w-1\right]\left[A w^{2}+B w+C\right]=0,
$$

where

$$
\begin{gathered}
A=\rho_{1}^{2} \rho_{2}^{2}(2 v-1)^{2}, \\
B=\rho_{1} \rho_{2}\left\{\nu(1-v)\left[1+\left(\rho_{1}+\rho_{2}\right)^{2}\right]\right. \\
\left.+\left[\rho_{1} v+\rho_{2}(1-v)\right]\left[1+(2 v-1)^{2}\right]\right\} \\
C=v(1-v)\left[\rho_{1}+\rho_{2}(1-v)\right]\left[1+\left(\rho_{1}+\rho_{2}\right)^{2}\right] \\
+\left[\rho_{1} v+\rho_{2}(1-v)\right]^{2}+\left(\rho_{1}-\rho_{2}\right)^{2} v^{2}(1-v)^{2} .
\end{gathered}
$$

Here $A, B, C$ have different meanings from those in Section 4 . Clearly $A, B, C$ are all positive. If $v=1 / 2, A=0$ and then $w=-C / B<0$ is a root (A.11), but it cannot be a root of $\operatorname{den}(w)=0$.

If $v \neq 1 / 2, A w^{2}+B w+C=0$ has no roots where $w \geqslant$ 0 , but we can show that it has two real roots in the range $w<0$. Letting $B=\rho_{1} \rho_{2} B_{0}$ and $A=\rho_{1}^{2} \rho_{2}^{2} A_{0}$ we must show 
that the discriminant DISC $\equiv B_{0}^{2}-4 A_{0} C>0$. After some calculations we find that

$$
\begin{array}{r}
\text { DISC }=v^{2}(1-v)^{2}\left[\left(1+a^{2}\right)^{2}+8 e\left(1+a^{2}\right)\right. \\
\left.+16 e^{2}-4(2 v-1)^{2} b^{4}\right],
\end{array}
$$

where

$$
e=\rho_{1} v+\rho_{2}(1-v) .
$$

But $\left(1+a^{2}\right)^{2}>4 a^{2}=4\left(\rho_{1}+\rho_{2}\right)^{2} \geqslant 4\left(\rho_{1}-\rho_{2}\right)^{2} \geqslant 4(1-$ $2 v)^{2}\left(\rho_{1}-\rho_{2}\right)^{2}=4(1-2 v)^{2} b^{4}$, since $v \in(0,1)$. Thus DISC $>0$ for $v \in(0,1)$ and the last factor in (A.11) has two distinct negative roots. If $v=0$ or $v=1$, there is a double root in the range $w<0$, as then DISC $=0$. In either case these negative roots cannot be roots of den $(w)$.

We have shown that any root of $\operatorname{den}(w)$ must be a root of (A.11), and this quartic has only real roots. Then only one $w=1$ and $w=\left(\rho_{1}+\rho_{2}\right)^{-2}$ can be roots of $\operatorname{den}(w)$, and we already showed that the former is always a root while the latter is a root if and only if $\left(\rho_{1}+\rho_{2}\right)^{2}>\rho_{1}-\rho_{2}$. Note that

$$
\begin{aligned}
\Delta_{1}\left(w_{p}\right) & =\left(1+\rho_{1}+\rho_{2}\right)^{2}-4 \rho_{1}-\frac{4 \rho_{1} \rho_{2}}{\left(\rho_{1}+\rho_{2}\right)^{2}} \\
& =\frac{1}{\left(\rho_{1}+\rho_{2}\right)^{2}}\left[\left(\rho_{1}+\rho_{2}\right)^{2}-\left(\rho_{1}-\rho_{2}\right)\right]^{2}, \\
\Delta_{2}\left(w_{p}\right) & =\frac{1}{\left(\rho_{1}+\rho_{2}\right)^{2}}\left[\left(\rho_{1}+\rho_{2}\right)^{2}-\left(\rho_{2}-\rho_{1}\right)\right]^{2}
\end{aligned}
$$

so that for $\rho_{1}>\rho_{2}, \sqrt{\Delta_{2}\left(w_{p}\right)}$ is always positive, while $\sqrt{\Delta_{1}\left(w_{p}\right)}$ is proportional to $\left|\left(\rho_{1}+\rho_{2}\right)^{2}-\left(\rho_{1}-\rho_{2}\right)\right|$, which vanishes along the transition curve.

\section{Acknowledgments}

Chalres Knessl work was partly supported by NSA Grant $\mathrm{H}$ 98230-11-1-0184. Haishen Yao was supported by PSC-CUNY research award no. 64349-0042.

\section{References}

[1] L. Flatto, "The longer queue model," Probability in the Engineering and Informational Sciences, vol. 3, pp. 537-559, 1989.

[2] Y. S. Zheng and P. Zipkin, "A queueing model to analyze the value of centralized inventory information," Operations Research, vol. 38, pp. 296-307, 1990.

[3] J. W. Cohen, "A two-queue, one-server model with priority for the longer queue," Queueing Systems. Theory and Applications, vol. 2, no. 3, pp. 261-283, 1987.

[4] L. Flatto and H. P. McKean, "Two queues in parallel," Communications on Pure and Applied Mathematics, vol. 30, no. 2, pp. 255-263, 1977.

[5] I. J.-B. F. Adan, J. Wessels, and W. H. M. Zijm, "Analysis of the symmetric shortest queue problem," Communications in Statistics. Stochastic Models, vol. 6, no. 4, pp. 691-713, 1990.
[6] I. J.-B. F. Adan, J. Wessels, and W. H. M. Zijm, "Analysis of the asymmetric shortest queue problem," Queueing Systems. Theory and Applications, vol. 8, no. 1, pp. 1-58, 1991.

[7] L. Flatto and S. Hahn, "Two parallel queues created by arrivals with two demands-I," SIAM Journal on Applied Mathematics, vol. 44, no. 5, pp. 1041-1053, 1984.

[8] L. Flatto, "Two parallel queues created by arrivals with two demands-II," SIAM Journal on Applied Mathematics, vol. 45, no. 5, pp. 861-878, 1985.

[9] G. Fayolle and R. Iasnogorodski, "Two coupled processors: the reduction to a Riemann-Hilbert problem," Zeitschrift für Wahrscheinlichkeitstheorie und Verwandte Gebiete, vol. 47, no. 3, pp. 325-351, 1979.

[10] A. G. Konheim, I. Meilijson, and A. Melkman, "Processorsharing of two parallel lines," Journal of Applied Probability, vol. 18, no. 4, pp. 952-956, 1981.

[11] F. Guillemin and D. Pinchon, "Analysis of generalized processor-sharing systems with two classes of customers and exponential services," Journal of Applied Probability, vol. 41, no. 3, pp. 832-858, 2004.

[12] F. Guillemin and J. S. H. van Leeuwaarden, "Rare event asymptotics for a random walk in the quarter plane," Queueing Systems. Theory and Applications, vol. 67, no. 1, pp. 1-32, 2011.

[13] J. W. Cohen, "Boundary value problems in queueing theory," Queueing Systems. Theory and Applications, vol. 3, no. 2, pp. 97128, 1988.

[14] J. W. Cohen and O. J. Boxma, Boundary Value Problems in Queueing System Analysis, vol. 79 of North-Holland Mathematics Studies, North-Holland Publishing Co., Amsterdam, The Netherlands, 1983.

[15] G. Fayolle, R. Iasnogorodski, and V. Malyshev, Random Walks in the Quarter-Plane, vol. 40, Springer, Berlin, Germany, 1999.

[16] N. I. Muskhelishvili, Singular Integral Equations, Dover, New York, NY, USA, 1992.

[17] R. Wong, Asymptotic Approximations of Integrals, vol. 34, SIAM, Philadelphia, Pa, USA, 2001.

[18] N. Bleistein and R. A. Handelsman, Asymptotic Expansions of Integrals, Dover, New York, NY, USA, 2nd edition, 1986.

[19] C. M. Bender and S. A. Orszag, Advanced Mathematical Methods for Scientists and Engineers, McGraw-Hill Book, New York, NY, USA, 1978.

[20] P. Flajolet and R. Sedgewick, Analytic Combinatorics, Cambridge University Press, Cambridge, UK, 2009.

[21] W. Szpankowski, Average Case Analysis of Algorithms on Sequences, Wiley-Interscience, New York, NY, USA, 2001.

[22] H. Yao and C. Knessl, "On the shortest queue version of the Erlang loss model," Studies in Applied Mathematics, vol. 120, no. 2, pp. 129-212, 2008.

[23] H. Yao and C. Knessl, "On the finite capacity shortest queue problem," Progress in Applied Mathematics, vol. 2, pp. 1-34, 2011. 


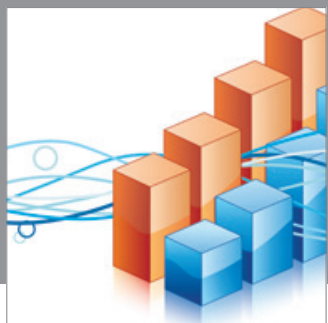

Advances in

Operations Research

mansans

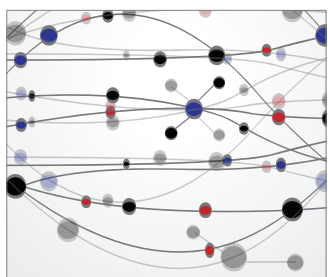

The Scientific World Journal
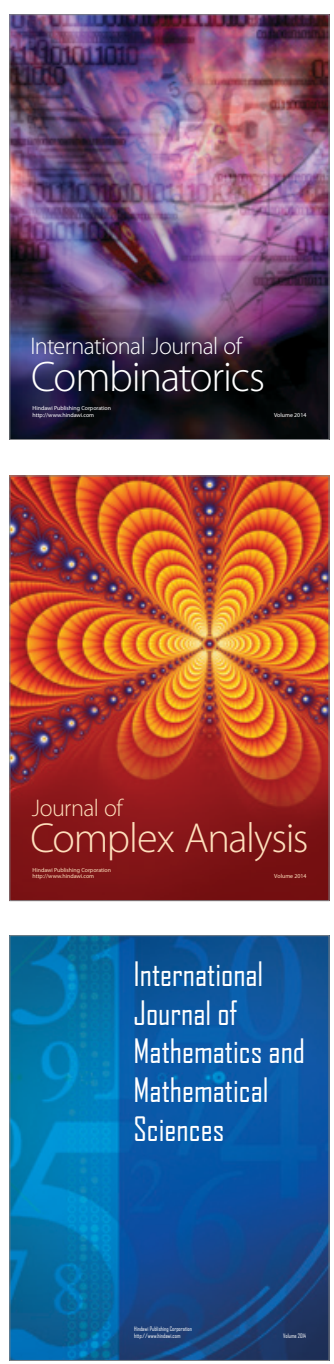
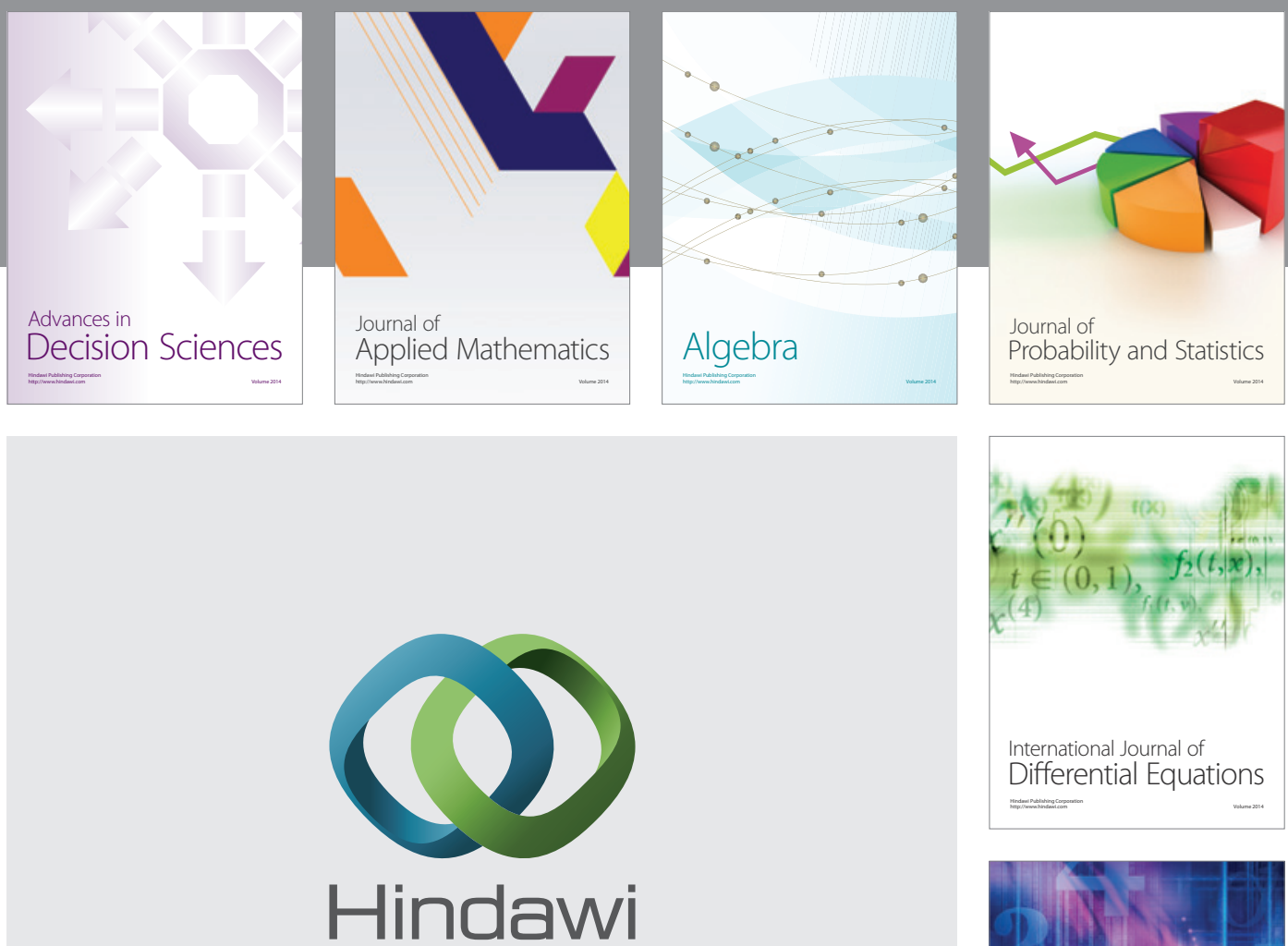

Submit your manuscripts at http://www.hindawi.com
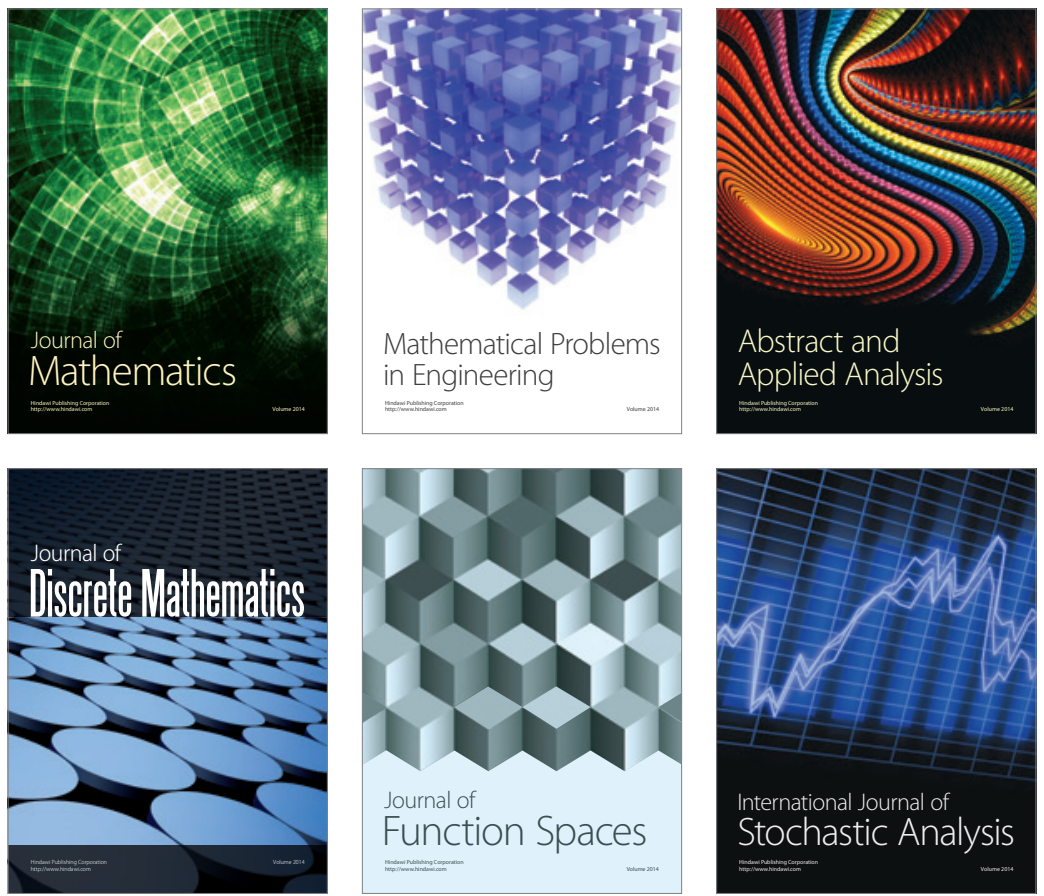

Journal of

Function Spaces

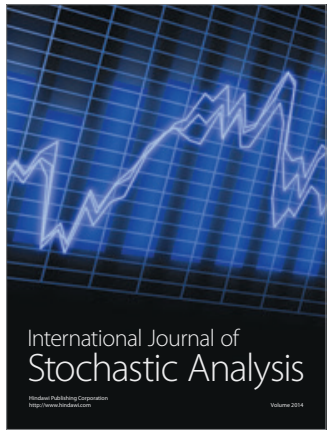

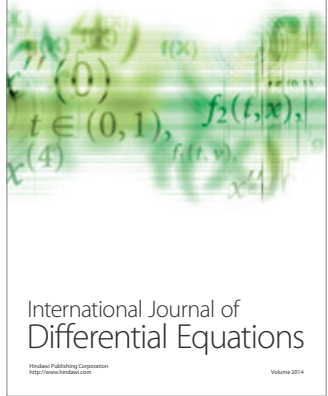
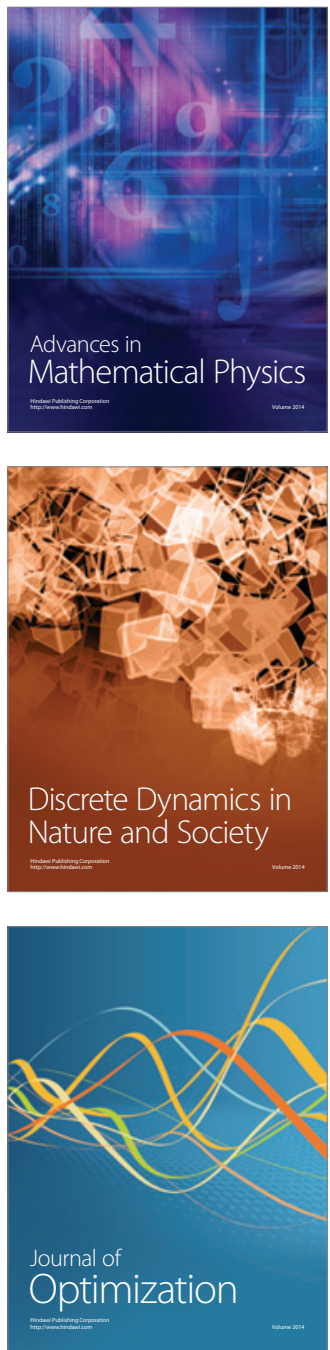